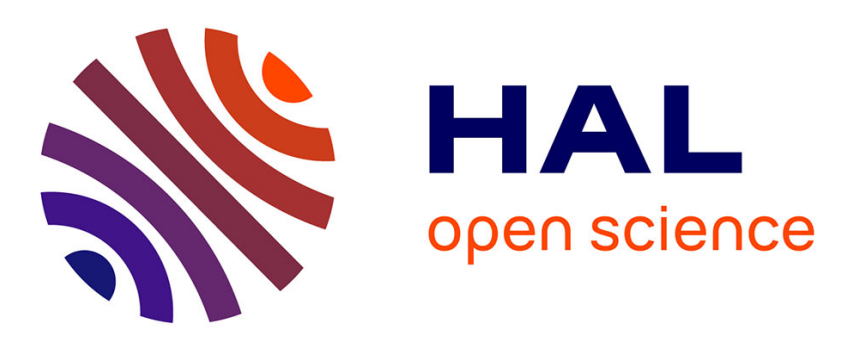

\title{
A Context-Oriented Framework for Computation Offloading in Vehicular Edge Computing using WAVE and 5G Networks
}

\author{
Alisson Barbosa de Souza, Paulo Antonio Leal Rego, Tiago Carneiro, Paulo \\ Henrique Gonçalves Rocha, José Neuman de Souza
}

\section{To cite this version:}

Alisson Barbosa de Souza, Paulo Antonio Leal Rego, Tiago Carneiro, Paulo Henrique Gonçalves Rocha, José Neuman de Souza. A Context-Oriented Framework for Computation Offloading in Vehicular Edge Computing using WAVE and 5G Networks. Vehicular Communications, 2021, 10.1016/j.vehcom.2021.100389 . hal-03295264

\section{HAL Id: hal-03295264 \\ https://hal.science/hal-03295264}

Submitted on 21 Jul 2021

HAL is a multi-disciplinary open access archive for the deposit and dissemination of scientific research documents, whether they are published or not. The documents may come from teaching and research institutions in France or abroad, or from public or private research centers.
L'archive ouverte pluridisciplinaire HAL, est destinée au dépôt et à la diffusion de documents scientifiques de niveau recherche, publiés ou non, émanant des établissements d'enseignement et de recherche français ou étrangers, des laboratoires publics ou privés. 


\title{
A Context-Oriented Framework for Computation Offloading in Vehicular Edge Computing using WAVE and $5 \mathrm{G}$ Networks
}

\author{
Alisson Barbosa de Souza ${ }^{\mathrm{a}, *}$, Paulo Antonio Leal Rego ${ }^{\mathrm{a}}$, Tiago Carneiro ${ }^{b c}$, \\ Paulo Henrique Gonçalves Rocha ${ }^{a}$, José Neuman de Souza ${ }^{a}$ \\ ${ }^{a}$ GREat - Federal University of Ceará, Fortaleza, Brazil \\ ${ }^{b}$ University of Luxembourg, Belval, Luxembourg \\ ${ }^{c}$ INRIA Lille - Nord Europe, Lille, France
}

\begin{abstract}
Despite technological advances, vehicles are still unable to meet the demands of some applications for massive computational resources in a feasible time. One way to deal with this situation is to integrate the computation offloading technique into a vehicular edge computing system. This integration allows application tasks to be executed on neighboring vehicles or edge servers coupled to base stations. However, the dynamic nature of vehicular networks, allied to overloaded servers, can lead to failures and reduce the effectiveness of the offloading technique. Therefore, we propose a context-oriented framework for computation offloading to reduce the application execution time and maintain high reliability in vehicular edge computing. The framework modules perform computational resources discovery, contextual data gathering, computation tasks distribution, and failure recovery. Its main part is a task assignment algorithm that seeks the best possible server to execute each application task, using contextual information and WAVE and 5G networks. The results of extensive experiments in different vehicular environments show that our framework reduces up to $70.3 \%$ of total execution time compared to totally local execution and up to $42.9 \%$

\footnotetext{
* Corresponding author

Email addresses: alisson@ufc.br (Alisson Barbosa de Souza), pauloalr@ufc.br (Paulo Antonio Leal Rego), tiago.carneiro-pessoa@inria.fr (Tiago Carneiro ${ }^{b}$ ), paulorocha@great.ufc.br (Paulo Henrique Gonçalves Rocha), neuman@ufc.br (José Neuman de Souza)
}

Preprint submitted to Vehicular Communications

July 21, 2021 
compared to other literature approaches. Concerning reliability, our framework achieves to offload up to $89.4 \%$ of all tasks and needs to recover only $0.8 \%$ of them. Thus, our solution outperforms the totally local execution of the application and other existing computation offloading solutions.

Keywords: Vehicular edge computing, Computation offloading, Task offloading, WAVE, 5G, Task assignment

\section{Introduction}

Vehicular technologies are increasingly advancing in terms of communication and intelligence. For example, Vehicular Ad Hoc Networks (VANETs) provide Vehicle to Vehicle (V2V) and Vehicle to Infrastructure (V2I) communications

5 [1, 2] using technologies such as Wireless Access in Vehicular Environments (WAVE)/IEEE 802.11p [3] and 5G/mmWave (millimeter Wave) [4, 5]. Vehicles have also evolved in intelligence through computing capabilities, cameras, embedded systems, sensors, and satellite navigation systems [6].

However, the advent of autonomous vehicles and new and popular applications such as augmented reality, automatic object recognition, and real-time video surveillance demand massive computing resources to deal with complicated data processing and critical latency requirements [7, [8. Thus, despite technological advances, vehicles do not yet have sufficient on-board computing resources to handle all of these requirements in a feasible time. Even if more powerful processors were installed in the vehicles, this could compromise their energy and displacement efficiency [6].

One manner to assist vehicles with latency and processing requirements is the vehicular edge computing system. In this system, computational processing can be done on Vehicular Clouds (VCs) or edge servers in an integrated or isolated 20 way, as can be seen in Figure 1. In turn, vehicular clouds are a pool of computational resources of two or more vehicles, stationary or in motion, which can be dynamically coordinated to offer services on demand, through V2V connections and on-board units (OBUs), as in the cloud computing model. Although 
VCs have less latency in communication and operate in scenarios without infrastructure, they do not present great computational power. Another option is to use edge servers coupled with Roadside Units (RSUs) or base stations (BSs) through V2I connections. These servers are deployed close to streets and roads by service providers. Even though they have a little more communication latency and can be quite requested by network devices, these servers generally have greater computational capacity than vehicular clouds 9 .

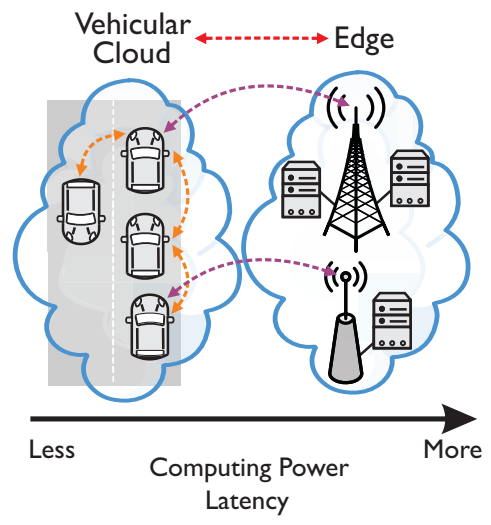

Figure 1: Vehicular edge computing (red line) system showing the integration between the vehicular cloud and the edge and aspects of latency and computing power. Orange lines represent V2V connections. Purple lines represent V2I connections.

One way to take advantage of these available computing resources is to apply the computation offloading technique, also called task offloading. This technique offloads smaller parts or tasks of an application to remote devices or servers, which are vehicles or edge servers in vehicular edge computing systems. Then, these servers process the tasks and return the result to the client. Computation offloading is used to improve applications' execution time and decrease the overload of processing [10, 11]. In fact, one of the main objectives of the computation offloading technique is to allow an application to execute in parallel in less time than execution on the client only [7].

40 Nevertheless, computation offloading is not always worthwhile due to communication and processing delays of remote servers [7. Moreover, the dynamic 
nature of vehicular networks can reduce the effectiveness of the offloading technique. This is because the network nodes have rapid movements, leading to frequent disconnections and offloading failures. Additionally, the servers may already be overloaded, and there is no support from a central coordination point [2]. In such cases, contextual information helps to deal with these challenges. Context refers to information that characterizes entities, including the situation of network devices or vehicles. Although contextual information changes dynamically, it assists in decision or adaptation processes and improves the performance of offloading systems [11, 12. For example, the information about the complete trajectory or route of a vehicle, if available, enables more accurate vehicle positioning predictions and helps to deliver data between network nodes [13].

Therefore, we propose a context-oriented framework for computation offloading in vehicular edge computing systems using WAVE and 5G networks. The objective is to ensure the reduction of application execution time and the reduction of offloading failures. The framework modules help the vehicle to discover computational resources and gather contextual data from devices within its communication range. Such information is used by the algorithm of decision and task assignment called Greedy Task by Task (GTT), the main part of the framework. GTT decides whether offloading is worthwhile and, if so, decides where to send the tasks. This decision is also the most challenging part of the framework because finding the best way to distribute tasks to minimize application execution time is an NP-hard problem 9]. For this decision, we consider contextual information such as speed, location, direction, CPU capacity and availability, data rates and communication ranges WAVE and 5G, link lifetimes, tasks characteristics, distances between devices, transmission and processing times, connectivity, known routes of vehicles, and estimated arrival time to the destination. After the decision, the framework distributes the computation tasks. Besides, if any task is lost or not executed, the framework has mechanisms to recover from failures. 


\subsection{Challenging Issues}

Thus, the main challenges related to this work are summarized below:

- Adaptation of computation offloading to the fast movement of nodes in vehicular networks. This behavior of nodes is linked to frequent disconnections, causing offloading failures [2].

- Gathering of contextual information. In dynamic environments, this information needs to be gathered and treated in real-time to be valid [12.

- NP-hardness of the task assignment decision problem to minimize application execution time. In this way, there is no exact polynomial-time solution for this problem. Furthermore, this decision needs to consider different contextual parameters 9 .

\subsection{Contributions}

We describe the main contributions of this work as follows:

- A context-oriented framework for computation offloading in vehicular edge computing with descriptions of the conceptual architecture and offloading and failure recovery processes.

- GTT, a task assignment algorithm that seeks the best possible servers for each application task, aiming to minimize applications' execution time and process failures. For this, various parameters of contextual information are taken into account.

- Use of a special contextual information about known routes of vehicles, helping to predict vehicle positioning more accurately and avoid offloading failures.

- Simultaneous use of WAVE and 5G technologies, combining their advantages, increasing capacities, and decreasing task transmission delays. 
- Extensive simulations of the proposed solution and literature algorithms, making it possible to evaluate and validate them in different vehicular environments in terms of execution time and reliability.

\subsection{Organization}

The remainder of the manuscript is organized as follows. Section 2 presents related works. An overview of the system model and problem formulation is given in Section 3. Section 4 describes the proposed framework, detailing its conceptual architecture and processes and its task assignment algorithm. The details of the experiments are presented in Section 5. Section 6 discusses the results. Finally, Section 7 presents the conclusions of this work.

Moreover, we have provided a list of acronyms definitions in Table 1 to make the paper easier to read.

Table 1: Acronyms definitions.

\begin{tabular}{llll}
\hline ALPR & Automatic License Plate Recognition & TL & Task executed locally \\
BS & Base Station & TR & Task executed with recovery \\
FIFO & First In, First Out & TS & Task successfully executed remotely \\
GCF & Greedy for CPU Free & V2I & Vehicle to Infrastructure \\
GTT & Greedy Task by Task & V2V & Vehicle to Vehicle \\
HVC & Hybrid Vehicular edge Cloud & VANET & Vehicular Ad Hoc Network \\
MDO & Multi-Decision based Offloading & VC & Vehicular Cloud \\
mmWave & Millimeter Wave & WAVE & Wireless Access in Vehicular Environments \\
RSU & Roadside Unit & &
\end{tabular}

\section{Related Works}

Several works have been developed in the area of computation offloading. Among these works, some consider that the clients (devices with applications that need computing offloading) are people's smartphones or virtual/augmented reality devices that participate in non-vehicular environments [14, 15, 16. Thus, the solutions proposed in these works do not consider important aspects that should be considered when the client is a vehicle, such as fast mobility, frequent 
network topology changes, and variations in wireless communication channels [17]. In [18], clients are smartphones of people inside vehicles that send tasks to be processed on traditional cloud servers. However, in this computation offloading process, the distinctive aspects of vehicular networks are also not considered, and the objectives do not include reducing the execution time of vehicular applications. In addition, clients have energy constraints that vehicles do not have, and communicating with traditional cloud servers can add high access latency, making applications with ultra-low latency requirements unfeasible [9].

Therefore, although there are different aspects considered in the offloading works, this section focuses specifically on the principal works of computation offloading in vehicular edge computing systems that have two essential characteristics. The first is that clients are only vehicles. The second is that the main objective of the proposed solutions is to reduce vehicular applications' execution time. These two characteristics are also essential in our work.

In these vehicular edge computing systems, edge servers coupled to base stations or vehicles can act as servers and execute third parties' tasks. These servers can receive tasks by different communication technologies such as WAVE, $4 \mathrm{G}$, or $5 \mathrm{G}$. In [19, 20], authors presented solutions to allow vehicles to execute computation tasks on selected neighboring vehicles over $5 \mathrm{G} / \mathrm{V} 2 \mathrm{~V}$ connections.

135 Nevertheless, using only one communication technology limits the capabilities to transmit more network packets and prevents connections to other types of networks [21, 22, 23]. Another interesting work in [24] proposed a computation offloading framework for $5 \mathrm{G}$ networks. The framework allows tasks to be executed only on edge servers coupled to base stations or RSUs. However, these proposed solutions allow third party tasks to be executed only by vehicles or only by edge servers. In either case, there is a waste of computational resources from unutilized servers.

Some works allow third-party tasks to be executed by vehicles and edge servers to avoid such a waste of resources. Besides, to increase the network's transmission capacities, these works use WAVE and 4G technologies. In [25, 26, 27, 28, proposed schemes and algorithms enable a vehicle to transfer its 
computations tasks to neighboring vehicles (via WAVE/V2V) or nearby edge servers (via 4G/V2I). Then, the latter can process the tasks and return their results to the requesting vehicle. Nonetheless, while it is an interesting approach, $4 \mathrm{G}$ networks are already starting to become outdated. They have deficiencies in relation to $5 \mathrm{G}$ networks, such as lower data rates, weaker for high mobility, fewer frequency ranges, and higher latencies. Also, algorithms used in 4G networks may not be well adapted to $5 \mathrm{G}$ networks because of differences in network architecture, modulation and multiplexing techniques, wireless signal behavior, limited range, and need for a line of sight between devices [29, 30, 31, 4,

In 32 , the authors proposed a framework to take advantage of several computational resources available locally, in nearby vehicles (via V2V), and on edge servers (via V2I). WAVE, 4G, and 5G technologies can also be used to increase network capacity. Even so, there is a prioritization of sending tasks via 5G. In scenarios where only base stations and few vehicles use $5 \mathrm{G}$, the sending of tasks will end up being, mostly, only via V2I and to the edge servers. Thus, this prioritization by $5 \mathrm{G}$ can overload the edge servers and underutilize the vehicles' computational resources. Moreover, sending via $5 \mathrm{G}$ can result in failures due to inherent technology challenges, such as limited range and the need for devices to be in the same line of sight. Also, if $5 \mathrm{G}$ fails, the algorithm in 32 will need to seek for resources via WAVE, which ends up generating more delays in the offloading process.

Even using different technologies, a computation offloading solution can work well in one scenario and not work in another. For example, scenarios with many intersections of streets (urban) or a single road (highway) influence network connectivity and interfere with the proposed solutions' performance. This also occurs with different vehicular densities [9]. Accordingly, it is important to submit the proposed solutions to different scenarios and densities, providing credibility to them, and evaluating the impact of specific situations. Despite this, as summarized in Table 2, none of these previous works used both the highway and urban scenarios in the experiments. Furthermore, most did not use all the main types of vehicular densities. 
Table 2: Comparison of different aspects used in related works.

\begin{tabular}{|c|c|c|c|c|c|c|c|c|c|c|c|c|c|}
\hline \multirow[t]{2}{*}{ Reference } & \multicolumn{2}{|c|}{ Technology } & \multicolumn{2}{|c|}{ Server } & \multicolumn{2}{|c|}{ Scenario } & \multicolumn{3}{|c|}{ Vehicular Density } & \multicolumn{3}{|c|}{ Contextual Information } & \multirow{2}{*}{$\begin{array}{c}\text { Objective } \\
+ \text { Reliability }\end{array}$} \\
\hline & WAVE & Cellular & $\mathrm{VC}$ & Edge & Urban & Highway & High & Medium & Low & $\begin{array}{l}\text { Known } \\
\text { Routes }\end{array}$ & $\begin{array}{c}\text { CPU } \\
\text { Capacity }\end{array}$ & $\begin{array}{c}\text { CPU } \\
\text { Availability }\end{array}$ & \\
\hline Ref. [19] & & $5 \mathrm{G}$ & $\sqrt{ }$ & & $\sqrt{ }$ & & & & & & $\sqrt{ }$ & $\sqrt{ }$ & $\sqrt{ }$ \\
\hline Ref. [20] & & $5 \mathrm{G}$ & $\sqrt{ }$ & & & $\sqrt{ }$ & & & $\sqrt{ }$ & & $\sqrt{ }$ & & \\
\hline Ref. 24] & $\sqrt{ }$ & $5 \mathrm{G}$ & & $\sqrt{ }$ & $\sqrt{ }$ & & & & $\sqrt{ }$ & & $\sqrt{ }$ & $\sqrt{ }$ & \\
\hline Ref. 25] & $\sqrt{ }$ & $4 \mathrm{G}$ & $\sqrt{ }$ & $\sqrt{ }$ & & $\sqrt{ }$ & & & $\sqrt{ }$ & & $\sqrt{ }$ & & \\
\hline Ref. 26] & $\sqrt{ }$ & $4 \mathrm{G}$ & $\sqrt{ }$ & $\sqrt{ }$ & & $\sqrt{ }$ & & & & & $\sqrt{ }$ & & \\
\hline Ref. 27] & $\sqrt{ }$ & $4 \mathrm{G}$ & $\sqrt{ }$ & $\sqrt{ }$ & & $\sqrt{ }$ & & & $\sqrt{ }$ & & $\sqrt{ }$ & & \\
\hline Ref. 28] & $\sqrt{ }$ & $4 \mathrm{G}$ & $\sqrt{ }$ & $\sqrt{ }$ & & $\sqrt{ }$ & & $\sqrt{ }$ & $\sqrt{ }$ & & $\sqrt{ }$ & $\sqrt{ }$ & \\
\hline Ref. 32] & $\sqrt{ }$ & $4 \mathrm{G} / 5 \mathrm{G}$ & $\sqrt{ }$ & $\sqrt{ }$ & $\sqrt{ }$ & & & & $\sqrt{ }$ & & $\sqrt{ }$ & $\sqrt{ }$ & \\
\hline Ref. 33] & $\sqrt{ }$ & $5 \mathrm{G}$ & $\sqrt{ }$ & $\sqrt{ }$ & & $\sqrt{ }$ & $\sqrt{ }$ & $\sqrt{ }$ & $\sqrt{ }$ & & & $\sqrt{ }$ & $\sqrt{ }$ \\
\hline Our proposal & $\sqrt{ }$ & $5 \mathrm{G}$ & $\sqrt{ }$ & $\sqrt{ }$ & $\sqrt{ }$ & $\sqrt{ }$ & $\sqrt{ }$ & $\sqrt{ }$ & $\sqrt{ }$ & $\sqrt{ }$ & $\sqrt{ }$ & $\sqrt{ }$ & $\sqrt{ }$ \\
\hline
\end{tabular}

In order to help deal with different scenarios, contextual information provides data that assists computation offloading systems [11]. One such information is the known routes of vehicles, a set of geographical coordinates that vehicles will travel. This information helps estimate vehicles' positions within a given time more accurately, avoiding sending tasks to those who will lose connectivity [13]. In our previous work [33, we proposed a scheme that applies computation offloading to edge servers, via 5G/V2I, and vehicles, via WAVE/V2V. However, the scheme does not use information about CPU capacity and known routes of vehicles. In fact, although the works mentioned in this section use various contextual information, none of them use the known routes of vehicles. Moreover, most of the works in Table 2 does not aim to increase reliability by counting and recovering eventual failures, even knowing that a failure can lead to increased latency, incomplete information, and application crashes.

Our proposed framework aims to fill the gaps left by the mentioned works. In terms of technology and servers, we allow tasks to be sent to edge servers and vehicles simultaneously through $5 \mathrm{G} / \mathrm{V} 2 \mathrm{I}$ and WAVE/V2V connections. With this, we increase the network's transmission capacities and took advantage of all available computing resources. In addition, we use several contextual in- 
formation, such as CPU capacity and availability, distances between devices, WAVE and 5G ranges, known routes of vehicles, and estimated arrival time to the destination. Then, we use an algorithm to assign tasks so that the best available servers execute them, aiming to minimize the applications' execution time and the number of offloading failures. Finally, we used different scenario configurations to analyze our proposal.

For more details about other works, we have done an extensive review and classification of several solutions of computation offloading in VANETs 9 .

\section{System Model and Problem Formulation}

This section presents an overview of the system model and problem formulation. First, we describe the network general structure. Then, we present the models for communication and computation. Finally, we present the problem formulation. In turn, Table 3 lists the definitions of the main symbols used.

Table 3: Symbols definitions.

\begin{tabular}{|llll|}
\hline$\tau$ & Task & $r$ & Transmission rate \\
$B$ & Bandwidth & $S$ & Set of chosen servers \\
$C$ & Computational capacity & $s$ & Data size \\
$c$ & CPU cycles required to process a task & server & Chosen server for the client \\
client & Client vehicle & $T$ & Workload or set of tasks \\
$d$ & Distance & $t$ & Time \\
$F$ & Set of feasible servers & $u$ & Velocity \\
$n_{\tau}$ & Total number of tasks in a workload & $W$ & Number of tasks to be executed by client \\
$P$ & Transmission power & $X$ & Number of tasks to be executed by servers \\
$p$ & Position & $Y$ & Backup of $T$ \\
$R$ & Communication range & & \\
\hline
\end{tabular}

\subsection{Network General Structure}

The network topology considered in this work has different types of nodes.

For example, it has a set of vehicles $V=\left\{v_{1}, v_{2}, v_{3}, \ldots, v_{n_{v}}\right\}$, where $n_{v}$ is the total number of vehicles on the network. The topology also has a set of edge servers $E=\left\{e_{1}, e_{2}, e_{3}, \ldots, e_{n_{e}}\right\}$, where $n_{e}$ is the total number of edge servers 
present on the network. Since each edge server is connected to a different $5 \mathrm{G}$ transmit something to the edge server, it must first transmit to the $5 \mathrm{G}$ base station. The latter then forwards the data to the edge server via a wired link. If the edge server wants to transmit something to a vehicle, the reverse path is 


\subsection{Communication Model}

Below, we present the communication models related to the link lifetime, position prediction based on known routes of vehicles, and data transmission rate.

\subsubsection{Link Lifetime}

It is possible to estimate how long two neighboring network nodes remain connected within each other's communication range. The estimate is made through kinematic calculations since parameters such as speed, direction, and distance between nodes do not vary significantly [34]. The work in [35] proposed a way to calculate this estimate. For this, we use four parameters: $p_{x}$ position of the node on the x-axis, $p_{y}$ position of the node on the y-axis, $u_{x}$ vector velocity of the node on the x-axis, and $u_{y}$ vector velocity of the node on the y-axis. We assume that the nodes follow a linear path in a short period. Thus, Equation 1 describes the position of the node $i$ as a function of time $t$ :

$$
p_{i}(t)=\left[\begin{array}{l}
p_{x_{i}}+u_{x_{i}} \cdot t \\
p_{y_{i}}+u_{y_{i}} \cdot t
\end{array}\right],
$$

where $p_{i}(t)$ is the position of node $i$ at time $t$.

We consider that a node $j$ is a neighbor of the node $i$. Thus, the Equation 2 shows how to estimate the future distance between nodes $i$ and $j$ :

$$
\begin{aligned}
d_{i, j}^{2} & =d_{j, i}^{2}=\left\|p_{j}(t)-p_{i}(t)\right\|^{2}=\left(\left[\begin{array}{l}
p_{x_{j}}-p_{x_{i}} \\
p_{y_{j}}-p_{y_{i}}
\end{array}\right]+\left[\begin{array}{l}
u_{x_{j}}-u_{x_{i}} \\
u_{y_{j}}-u_{y_{i}}
\end{array}\right] \cdot t\right)^{2} \\
& =\alpha_{i, j} t^{2}+\beta_{i, j} t+\gamma_{i, j},
\end{aligned}
$$

where $\alpha_{i, j} \geq 0, \gamma_{i, j} \geq 0$. Then, the future relative distance between $j$ and $i$ is $d_{i, j}(t)=\sqrt{\alpha_{i, j} t^{2}+\beta_{i, j} t+\gamma_{i, j}}$.

With this, it is possible to calculate the link lifetime. According to 35] and [36], the link lifetime of the two nodes $(i$ and $j$ ) is the estimated time 
$t_{\text {link }}^{i, j}=t_{1}-t_{0}$, where $t_{1}$ is the time when the distance $d$ between the two nodes becomes greater than their communication range $R$ and $t_{0}$ is the initial time of the nodes. For the two nodes to be connected, $d$ must be less than or equal to $270 \quad R$.

For $t_{\text {link }}^{i, j}$ to be calculated, it is necessary to make $d_{i, j}(t) \leq R$. If we square both sides of the inequality and put $R$ on the other side, we get the Equation 3. which gives the value of $t_{\text {link }}^{i, j}$ :

$$
d_{i, j}^{2}(t)-R^{2}=0
$$

Since we already have an equation that describes $d_{i, j}^{2}(t)$, we get the Equation 2754 below:

$$
\alpha_{i, j} t^{2}+\beta_{i, j} t+\gamma_{i, j}-R^{2}=0,
$$

where $t$ represents $t_{\text {link }}^{i, j}$. Thus, to know the value of $t_{\text {link }}^{i, j}$, it is only necessary to solve the second degree equation presented in Equation 4.

However, if two nodes have very similar mobility (for example, if they are close to each other, with similar velocities and going in the same direction), $t_{\text {link }}$ tends to be infinite. To adjust this question, 37. proposed an upper limit constant for very large values of $t_{\text {link }}$. We defined this constant as $t_{\text {maxlifetime }}$ with a value of 100 seconds. That way, if $t_{l i n k}>100 s, t_{l i n k}$ becomes 100 seconds.

\subsubsection{Position Prediction Based on Known Routes of Vehicles}

Public or private vehicles can share information from their on-board navigation systems, such as their trajectories/routes and the estimated arrival time to the destination. With this, it is possible to estimate, with more precision, if two neighboring network nodes will still be connected after a given time $t_{\varkappa}[13$.

The trajectory or route of a vehicle $j$ is a set of points (geographic coordinates) that will be traveled by it as follows:

$\left[\left(p_{x_{j}}\left(t_{0}\right), p_{y_{j}}\left(t_{0}\right)\right) ;\left(p_{x_{j}}\left(t_{1}\right), p_{y_{j}}\left(t_{1}\right)\right) ; \ldots ;\left(p_{x_{j}}\left(t_{n}\right), p_{y_{j}}\left(t_{n}\right)\right)\right]$, 
where $\left(p_{x_{j}}\left(t_{0}\right), p_{y_{j}}\left(t_{0}\right)\right)$ represents the latitude and longitude of the vehicle at the initial time $t_{0}$ and $\left(p_{x_{j}}\left(t_{n}\right), p_{y_{j}}\left(t_{n}\right)\right)$ represents the latitude and longitude of the vehicle at the estimated arrival time $t_{n}$.

Thus, the distance to be travelled $\left(d_{\text {total }}\right)$ is calculated as follows:

$$
d_{\text {total }}=\sum_{\ell=1}^{n} \sqrt{\left(p_{x_{j}}\left(t_{\ell}\right)-p_{x_{j}}\left(t_{\ell-1}\right)\right)^{2}+\left(p_{y_{j}}\left(t_{\ell}\right)-p_{y_{j}}\left(t_{\ell-1}\right)\right)^{2}} .
$$

Therefore, the average vehicle speed is $\overline{u_{j}}=d_{\text {total }} /\left(t_{n}-t_{0}\right)$. Then, the estimated distance traveled by the vehicle $j$ in a given time $t_{\varkappa}$ is: $d_{e s t}=\overline{u_{j}}\left(t_{\varkappa}-t_{0}\right)$.

For simplicity, we call the estimated position $p_{j}\left(t_{\varkappa}\right)$ of $\left(\varphi_{x}, \varphi_{y}\right)$. If $t_{\varkappa}>t_{n}$, then $\left(\varphi_{x}, \varphi_{y}\right)$ it is the final position of the trajectory, already known. If $t_{\varkappa}<t_{n}$, to find $\left(\varphi_{x}, \varphi_{y}\right)$, add the distances between the points of the vehicle's trajectory, from the initial point as in Equation 5 , until the sum of these distances is greater than $d_{e s t}$. When this happens, we know that $\left(\varphi_{x}, \varphi_{y}\right)$ is approximately on the line between the last and the penultimate points added, respectively $\left(\varpi_{x}, \varpi_{y}\right)$ and $\left(\varpi_{x-1}, \varpi_{y-1}\right)$. With these two points, we calculate the general equation of the line as a function of $\left(\varphi_{x}, \varphi_{y}\right)$ as follows:

$$
\left(\varpi_{y}-\varpi_{y-1}\right) \varphi_{x}+\left(\varpi_{x}-\varpi_{x-1}\right) \varphi_{y}+\varpi_{x} \varpi_{y-1}-\varpi_{x-1}-\varpi_{y}=0,
$$

In addition, the distance between $\left(\varphi_{x}, \varphi_{y}\right)$ and $\left(\varpi_{x-1}, \varpi_{y-1}\right)$ is $d_{e s t}$ minus the distance from the initial point of the vehicle's trajectory until $\left(\varpi_{x-1}, \varpi_{y-1}\right)$, which we call $d_{\varrho}$. Thus, we can obtain another equation as a function of $\left(\varphi_{x}, \varphi_{y}\right)$ :

$$
\sqrt{\left(\varphi_{x}-\varpi_{x-1}\right)^{2}+\left(\varphi_{y}-\varpi_{y-1}\right)^{2}}=d_{e s t}-d_{\varrho} .
$$

Combining the Equations 6 and 7, we were able to obtain the estimated position of the vehicle $j$ in time $\varkappa$. The same procedures are used to calculate the position of a vehicle $i$ in time $\varkappa$. After this, we calculate the distance between $i$ and $j$ in time $\varkappa$ and check if it is less than the communication range. 


\subsubsection{Data Transmission Rate}

According to [28, 26, 38, 39, in WAVE/V2V communications, the data transmission rate between a node $i$ and a node $j$ at a given time $t$ is given by:

$$
r_{V 2 V}^{i, j}(t)=B_{V 2 V} \log _{2}\left(1+\frac{P_{t} L_{i}^{V 2 V}\left|\phi^{2}\right|}{\omega}\right),
$$

where $B_{V 2 V}$ is the bandwidth between $i$ and $j, P_{t}$ is the transmission power of the node, $L_{i}^{V 2 V}$ is the loss of system propagation, $\phi$ is the fading coefficient of the uplink channel, and $\omega$ is the power of the white Gaussian noise.

Thus, the average uplink rate between nodes $i$ and $j$ is given by:

$$
\overline{r_{V 2 V}^{i, j}}=\frac{\int_{0}^{t_{l i n k}^{i, j}} r_{V 2 V}^{i, j}(t) \mathrm{d} t}{t_{l i n k}^{i, j}}
$$

As [40], for simplicity, we can neglect the time to access the control $(\mathrm{CCH})$ and service (SCH) WAVE channels and the time spent on switching channels, such as the guard interval.

Thus, the time it takes to transmit data of size $s$ from $i$ to $j$ via WAVE/V2V is given by:

$$
t_{\text {trans }}^{i, j}=\frac{s}{\overline{r_{V 2 V}^{i, j}}}+\frac{d^{i, j}}{u_{\text {prop }}}+t_{\text {other }},
$$

325

where $u_{\text {prop }}$ is the propagation speed over the wireless medium and $t_{\text {other }}$ are estimates of possible network and queue delays.

For $5 \mathrm{G} / \mathrm{V} 2 \mathrm{I}$ communications, the authors in [41, 42, 43] show that the data transmission rate between a node $i$ and a node $j$ in a given time $t$ is given by:

$$
r_{V 2 I}^{i, j}(t)=B_{V 2 I} \log _{2}\left(1+\frac{P_{t} d_{i, j}^{-\epsilon}\left|\phi^{2}\right|}{\omega}\right),
$$

where $B_{V 2 I}$ represents the bandwidth between $i$ and $j, d_{i, j}$ is the distance between $i$ and $j$ and the factor $\epsilon$ is the exponent of propagation loss of the system. Due to the fast transmission rates on the wired link and the coexisting 
deployment between the base station and the edge server, the transmission delay of the wired link is neglected in this work [43].

In this way, the average uplink rate between nodes $i$ and $j$ is given by:

$$
\overline{r_{V 2 I}^{i, j}}=\frac{\int_{0}^{t_{l i n k}^{i, j}} r_{V 2 I}^{i, j}(t) \mathrm{d} t}{t_{\text {link }}^{i, j}} .
$$
given by:

$$
t_{\text {trans }}^{i, j}=\frac{s}{\overline{r_{V 2 I}^{i, j}}}+\frac{d^{i, j}}{u_{\text {prop }}}+t_{\text {other }} .
$$

\subsection{Computation Model}

The vehicles have different computational capacities and CPU availability over time. The same is true with edge servers. Each vehicle or edge server has a computational capacity $C_{v}$ and $C_{e}$, respectively.

We consider a computationally intensive and real-time application that generates a workload or set $T=\left\{\tau_{1}, \tau_{2}, \tau_{3}, \ldots, \tau_{n_{\tau}}\right\}$ of tasks. Each task $\tau \in T$ can be processed locally by the client or remotely in an independent, asynchronous, and parallel way. Thus, each workload can have its tasks distributed for local 345 or remote execution (on the edge servers or in other vehicles) or in both local and remote environments. In addition, each task $\tau \in T$ is a tuple composed of the following parameters $\left\{c_{\tau}, s_{\tau}^{u p}, s_{\tau}^{\text {down }}\right\}$, where $c_{\tau}$ indicates the total number of CPU cycles required to execute the task $\tau, s_{\tau}^{u p}$ shows the data size for upload of $\tau$ (which includes input parameters, the code to be executed and information about the device that generated the task) and $s_{\tau}^{\text {down }}$ shows the size of the processing results of $\tau$ for download (which includes information on which device the results should be sent to). $s^{u p}$ and $s^{\text {down }}$ are known for the history of the application analyzed by the system. Each task is executed with $100 \%$ of the CPU available for task executions. Each CPU available from any node on the network can only execute one task at a time. Tasks are placed in a queue and taken out in First In, First Out (FIFO) model to be executed. 
Next, we can see the details of the computational modeling of the execution for each environment [28].

\subsubsection{Local Computation}

When the client chooses to execute a task locally, the local execution delay of the client is set to $t_{\text {client }}$. $C_{\text {client }}$ is described as the computational capacity of the client node (in CPU cycles per second). $t_{\text {client }}$ consists of two parts: 1 ) queue delay (there may be other tasks waiting to be executed or in execution) and 2) processing delay of the task on the CPU. The queue delay is given by:

$$
t_{\text {client }}^{q u e u e}=\sum_{w=1}^{g} \frac{c_{w}}{C_{\text {client }}},
$$

365 the number of tasks that were already waiting in the queue or executing.

The processing delay of a task $\tau$ on the client CPU is given by:

$$
t_{\text {client }}^{\text {proc }}=\frac{c_{\tau}}{C_{\text {client }}} .
$$

Thus, the local execution delay in the client node of a task $\tau \in T$ is given by:

$$
t_{\text {client }}=t_{\text {client }}^{\text {queue }}+t_{\text {client }}^{\text {proc }} .
$$

\subsubsection{Remote Server Computation}

A client vehicle generates a task and send it to execute on a remote server. As part of vehicular edge computing, the server is a vehicle or edge server. Such a server executes the task and returns the result of the execution to the client vehicle that generated the task.

375 Thus, the delay in executing the task on the server is divided into four parts. The first part is the upload of $s_{\tau}^{u p}$ from the client vehicle to the server. If the upload is via WAVE/V2V, the delay is given by Equation 10. If the upload is via $5 \mathrm{G} / \mathrm{V} 2 \mathrm{I}$, the delay is given by Equation 13 . 
The second part is the waiting time for the task in the server queue $t_{\text {server }}^{\text {queue }}$

380 waiting or executing on the server to finish executing. This time is given by:

$$
t_{\text {server }}^{\text {queue }}=\sum_{x=1}^{q} \frac{c_{x}}{C_{\text {server }}},
$$

where $x$ represents each task in the queue of the server node and $q$ represents the number of tasks that were already waiting in the queue or in execution.

The third part consists of the time it takes to process a task $\tau$ :

$$
t_{\text {server }}^{\text {proc }}=\frac{c_{\tau}}{C_{\text {server }}} .
$$

385

The fourth part of the delay is the time it takes to transmit the processing result $\left(s_{\tau}^{\text {down }}\right)$ from the server to the client. If the transmission is via WAVE/V2V, the time is given by Equation 10 If it is via 5G/V2I, the time is given by Equation 13 .

Thus, the delay for executing a task $\tau \in T$ on a remote server is given by:

$$
t_{\text {server }}=t_{\text {client }}^{\text {upload }}+t_{\text {server }}^{\text {queue }}+t_{\text {server }}^{\text {proc }}+t_{\text {client }}^{\text {download }} .
$$

\subsubsection{Total Execution Time}

As we consider that workload has a total of $n_{\tau}$ tasks, they are distributed to be executed locally and on remote servers (vehicles or edge servers).

So, we assume that tasks are distributed as follows:

- $W$ tasks are distributed to the client;

395

- $X_{1}$ tasks are distributed to the server $1, X_{2}$ to the server $_{2}, X_{3}$ to the server $_{3}$ and so on up to $X_{k}$ to the server $_{k}$, so that $X_{1}+X_{2}+X_{3}+\ldots+X_{k}=$ $X$

Thus, the total time required to execute tasks locally is given by:

$$
t_{\text {client }}^{\text {total }}=\sum_{w=1}^{W} t_{\text {client }}^{w}
$$


where $w$ represents each task distributed to the client node, and $W$ represents the total number of tasks distributed to the client.

In turn, the total time required to execute tasks on remote servers is given by:

$$
t_{\text {servers }}^{\text {total }}=\max \left\{\sum_{x_{1}=1}^{X_{1}} t_{\text {server }_{1}}^{x_{1}}, \sum_{x_{2}=1}^{X_{2}} t_{\text {server }_{2}}^{x_{2}}, \ldots, \sum_{x_{k}=1}^{X_{k}} t_{\text {server }_{k}}^{x_{k}}\right\}
$$

where $x_{1}$ represents each task distributed to the server $_{1}, x_{2}$ each task to the server $_{2}, x_{k}$ each task to the server $_{k} ; X_{1}$ represents the total number of tasks

distributed to the server $_{1}, X_{2}$ the total tasks to the server $_{2}$, and $X_{k}$ the total tasks to the server $_{k}$.

Therefore, the total time to execute the workload is described as:

$$
t^{\text {total }}=\max \left\{t_{\text {client }}^{\text {total }}, t_{\text {servers }}^{\text {total }}\right\}
$$

\subsection{Problem Formulation}

The objective of this work is to minimize the execution time of a vehicular application through the computation offloading process in vehicular edge computing systems, satisfying reliability restrictions. So the problem can be formulated as follows:

$$
\begin{aligned}
& P 1: \min t^{\text {total }} \\
& \text { s.t. } C 1: W+X=n_{\tau} \text {, } \\
& C 2: \sum_{x_{k}=0}^{X_{k}} t_{\text {server }_{k}}^{x_{k}}<t_{\text {link }}^{\text {client }, \text { server }_{k}} \\
& \vee\left(d_{\text {client }, \text { server }_{k}}\left(t_{\text {server }_{k}}^{x_{k}}\right)<R\right) \text {. }
\end{aligned}
$$

Thus, problem P1, identified as an NP-hard problem 9], involves finding a way to assign computation tasks to different servers in order to minimize $t^{\text {total }}$. ${ }_{415}$ The value of $t^{\text {total }}$ is calculated according to the Equation 22. This equation 
takes into account the total time required to execute tasks locally $\left(t_{\text {client }}^{\text {total }}\right)$ and the total time required to execute tasks on remote servers $\left(t_{\text {servers }}^{\text {total }}\right)$. The value

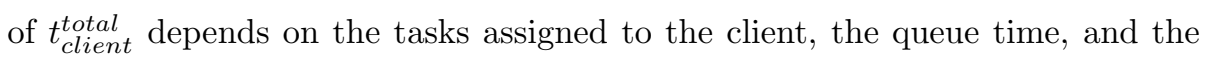
processing capacity of the client, as shown in Equation 20 and the equations in 420 Section 3.3 .1 . The value of $t_{\text {servers }}^{\text {total }}$ depends on the time to execute tasks on each server. In turn, this time depends on the tasks assigned, the processing capacity of the servers, their queue times, and upload and download times, as presented in Equations 19 and 21. The upload and download times follow the equations in Section 3.2.3. which consider the network bandwidth and tasks size, as well as other parameters.

Regarding the constraints of problem P1, C1 ensures that the sum of the total tasks executed on the client $(W)$ and the total tasks executed on remote servers $(X)$ is equal to the number of tasks in the application workload $\left(n_{\tau}\right)$. The constraint C2 means that the time $t_{\text {server }_{k}}^{x_{k}}$ to execute a workload $x_{k}$ on a 430 given server $_{k}$ needs to be less than the link lifetime between client and server $_{k}$, according to Equation 4 . Or, if the client and server $_{k}$ routes are known, the distance between their estimated positions in time $t_{\text {server }_{k}}^{x_{k}}$, following the Equations 6] and 7, must be less than the communication range $R$.

As mentioned before, the value of $t_{\text {server }}^{x_{k}}$ used in the C2 constraint is calcu435 lated according to the Equation 19. Such equation considers several parameters such as the characteristics of the tasks assigned, the bandwidth to transmit the tasks (see Section 3.2.3), the server's computational capacity, and its queue time (see Section 3.3.2). With the constraint C2, the problem formulation aims to contribute to greater reliability of the computation offloading process. This constraint makes the client aware of possible servers' mobility. It also prevents the client from choosing a possible server $_{k}$ that goes out of its communication range without completing the computation offloading process.

Although we consider several parameters in the problem formulation, we do not consider the storage capacity of devices in constraints for two reasons.

${ }_{445}$ The first is that the servers discard the tasks, freeing up storage space after proper processing and returning the results to the client. The second is that, 
unlike data offloading (or caching), the storage space required in computation offloading is typically small [45].

\section{Proposed Framework}

In this section, we present the proposed framework for vehicular systems. It manages all stages of the computation offloading technique to minimize the execution time of vehicular applications reliably, according to the objective and restrictions of problem P1. The main part of this framework, the task assignment algorithm, is responsible for providing good and feasible solutions to the problem at hand.

Next, we describe in detail the architecture of the proposed framework and its managed computation offloading process.

\subsection{Framework Architecture}

Figure 2 shows the Application and Partitioner modules and conceptual architecture of the proposed framework.

The Application module represents the applications running on the vehicle's operating system. As seen in Figure 2 , it sends data to a Partitioner module in order to analyze whether the application workload can be partitioned. If it is possible, the Partitioner divides the application workload into smaller tasks that can be executed on different devices and in a parallel, asynchronous, and independent way. The application workload then moves to the Decision Maker module. After the workload has been processed, the Application receives the results through the Local Execution or the Data and Context Gatherer module, when the results come from remote devices. Information from local sensors and other devices is also captured through the Data and Context Gatherer module.

Next, we describe each module of the proposed framework.

\subsubsection{Sensors}

The Sensors module is responsible for sending to the Data and Context Gatherer module local information such as location (via Global Positioning 


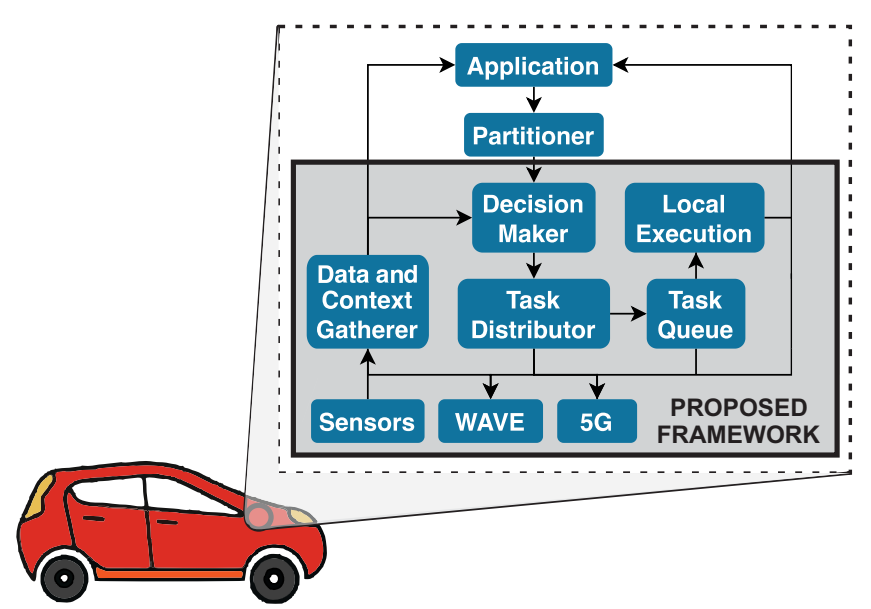

Figure 2: Application and Partitioner modules and conceptual architecture of the computation offloading framework proposed for vehicular systems. The box with dotted lines represents an application running on a vehicle, along with the Partitioner module and the proposed framework. The latter is represented by the smaller box with a gray background. The arrows indicate the direction of the information flow between the modules.

475 System), speed, and direction.

\subsubsection{Task Queue}

Tasks from remote devices or the client are placed in the Task Queue by the Task Distributor module. After a task passes through the queue, it goes to the Local Execution module. Information about the storage capacity of the queue and the number of tasks in it is periodically passed on to the Data and Context Gatherer module.

\subsubsection{Local Execution}

With CPU and other resources, the Local Execution module process tasks that come from the Task Queue with the processing delays described in Section 3.3 Through information contained in the task, this module checks whether it is local or came from a remote device. Then, it can forward the processing result to the local application or to remote devices (via the WAVE or $5 G$ modules). 


\subsubsection{WAVE and $5 G$}

The WAVE module is responsible for sending and receiving messages from the WAVE network via $\mathrm{V} 2 \mathrm{~V}$. The $5 G$ module is responsible for sending and receiving messages from the $5 \mathrm{G}$ network via V2I.

These modules can send periodic signaling and safety messages and other data to remote devices. Upon receiving messages, these modules forward them to the Data and Context Gatherer module. These messages can be: requests to execute a task to a remote device (offloading request), replies to offloading requests (offloading reply), tasks, data downloaded to a running application, and context data from other devices.

\subsubsection{Task Distributor}

This module analyzes the tasks received from the Decision Maker and the location where they will be executed. If the decision is to execute a task locally, the Distributor forwards it to the local Task Queue. If the decision is to run a task remotely, the Distributor sends it to the WAVE or $5 G$ modules (or both), depending on the choice made by the Decision Maker.

This module is also responsible for storing a backup of tasks that have been offloaded to remote servers. Thus, upon being informed that the remote server has failed to return the processing result, this module then sends the stored copies of the lost tasks to be executed locally.

\subsubsection{Data and Context Gatherer}

Through the WAVE and $5 G$ modules, the Data and Context Gatherer can receive messages involving application data or remote processing results. Then, the Gatherer forwards it to the local Application module. If the received message involves remote contextual information, this module forwards it to the Decision module. If the Gatherer receives periodic local contextual information through the Sensors, the Task Queue and the Local Execution module (CPU capacity), this module forwards it to the Application module or to the Decision module. Upon receiving an offloading request, a reply for an offloading request, or a task 
to be executed, this module forwards them to the Decision Maker module.

This module also monitors possible offloading failures. This is done by analyzing the signaling messages received from the chosen remote servers and verifying their connectivity with the client. After failure detection, the Gatherer notifies the Decision Maker that triggers the Task Distributor. More details of failure handling are described in Section 4.2.4.

\subsubsection{Decision Maker}

The Decision Maker module receives tasks from the Partitioner, starts resource discovery, and gathers all real-time contextual information (local and remote) from the Data and Context Gatherer. Then, the Decision Maker module calculates additional information such as link lifetime, distances between devices, transmission and processing time, and whether the client and server will be within the communication range of each other at a specific time.

With all this information, this module assigns, through a greedy algorithm (see Section 4.2.2), tasks for the chosen devices to execute. Subsequent, it informs the task assignment decision to the Task Distributor module.

This module also receives other types of messages. For example, when receiving offloading requests, the Decision Maker triggers the Distributor to reply to the requesting device agreeing to process its tasks. Upon receiving a positive reply for an offloading request, this module sends the tasks to the remote device through the Distributor and WAVE or $5 G$ module. Upon receiving a task to be executed, it forwards it to the local Task Queue, through the Distributor module.

\subsection{Computation Offloading Process}

This Section presents the computation offloading process managed by the proposed framework. The process consists of four main parts: 1) Resources discovery, 2) Offloading decision, 3) Send/receive tasks, and 4) Failure recovery. 


\subsubsection{Resources Discovery} receives tasks from the Application/Partitioner. Then, through the Distributor, WAVE and $5 G$ modules, it triggers the resources discovery. At this time, as shown in Figure 3, the client sends a one-hop WAVE request via broadcast to the other vehicles and sends a $5 \mathrm{G}$ request via unicast to the edge server within the respective communication ranges of each technology. These requests are used to gather real-time contextual information from possible servers and allow the Decision module to provide feasible solutions to problem P1.

Figure 3: Client (red) discovering computational resources of vehicles within its WAVE communication range (black dotted line) via V2V connections (blue dotted lines) and of an edge server whose base station is within its $5 \mathrm{G}$ communication range (light blue background) via V2I connection (purple dotted line). The client has three tasks to be executed, its computational capacity is low (pointer almost at least), and its task queue for execution is almost full (bar with small red blocks).

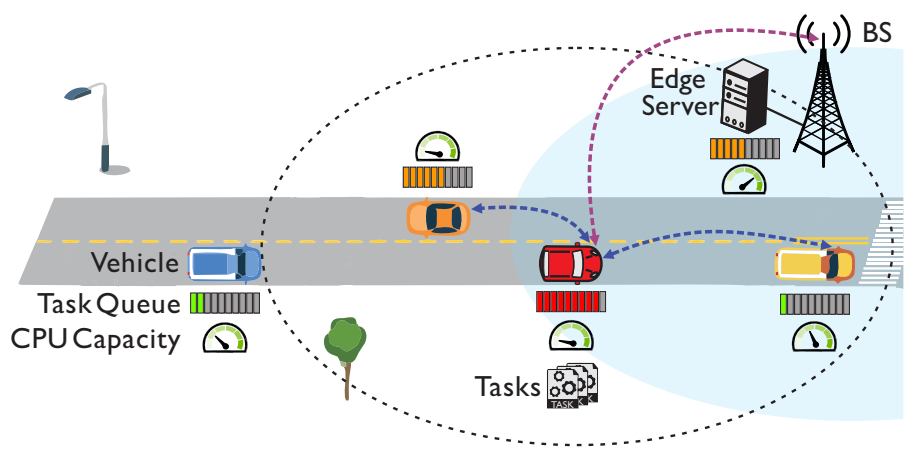

After the client's first contact, each possible server replies with a tuple containing its location, data rate and communication range (WAVE or 5G), CPU capacity, and task queue condition. If it is a vehicle, it also sends its speed, direction, and, if available, its estimated arrival time to the destination and its complete route. This reply reaches the client via the $W A V E$ and $5 G$ modules, which forwards it to the Data and Context Gatherer module, which in turn sends it to the Decision module. Subsequent waiting $\psi$ milliseconds, the 


\subsubsection{Offloading Decision}

The decision process is the core of the framework. This step is also the most complex because the optimal distribution of tasks for maximum reduction in application execution time is an NP-hard problem [9. Hence, no algorithm provides an exact solution for this type of problem in a polynomial time. Thus, we propose a greedy heuristic algorithm called Greedy Task by Task (GTT), which delivers reasonable solutions to the problem in a feasible time, although it does not guarantee to find the optimal solution [46].

After congregating all the necessary information, the Decision Maker modbuild task assignment solutions in stages considering the problem P1 and contextual information. Accordingly, the algorithm tries to minimize the execution time of each task by assigning them to the best possible server at the moment (local or remote). With this strategy, the GTT provides feasible solutions to 575 the problem P1. After finding a solution, the framework allows tasks to be sent simultaneously to edge servers (via 5G) and neighboring vehicles (via WAVE). We present the GTT's pseudocode in Algorithm 1.

GTT receives as input a set $M$ of reply messages and a set $T$ of tasks to be processed. In line 1, GTT initializes the sets $S$ (servers), $W$ (tasks to be executed locally), $Y$ (backup of $T$ ) and variable $i$ (client ID). In line 2 , the set $F$ of feasible servers is initialized with a tuple from the client, indicating that it can also execute tasks. Afterwards, in line $3, F$ receives more feasible servers on return from the Function AddFeasibleServers (Algorithm 2).

Then, in line 4 , the set $F$ of feasible servers is sorted in increasing order according to the distance to $i$, computational capacity, queue time, and the constants $\sigma, \rho$, and $\delta$. These parameters are some of the most used in computation offloading processes [7. In particular, the computational capacity and the queue time directly influence the task execution time. Distance is also important to avoid failures and delays due to retransmissions. In fact, the closer a server 590 is to the client, the less likely it is to experience failures caused by interference, 


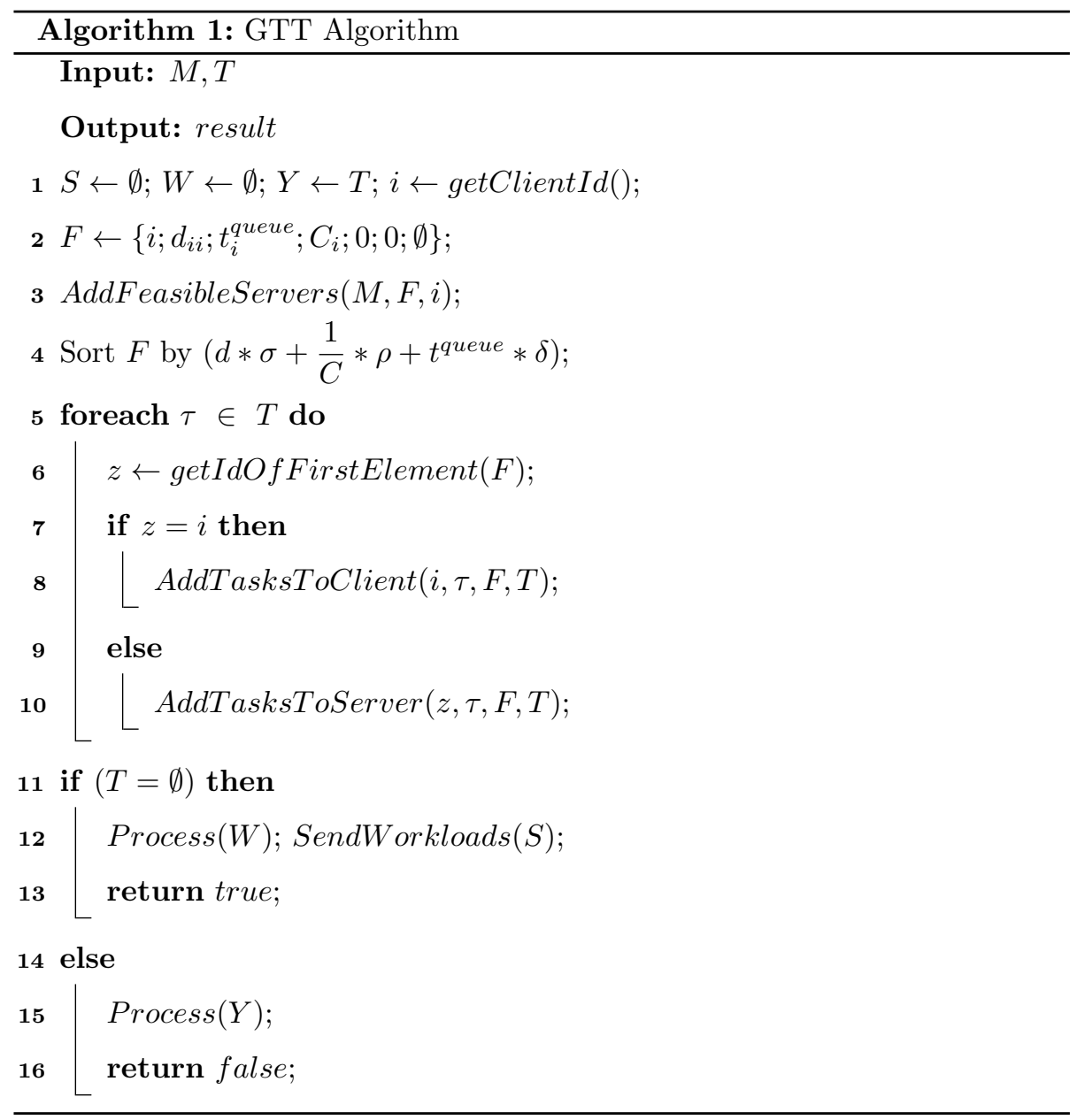

propagation loss, and 5G signal blocking [47, 48]. Thus, GTT prioritizes servers with high computational capacities, low queue times, and short distances to the client to minimize application execution time and avoid failures.

In loop of lines 5-10, $T$ is traversed task by task so that each task $\tau$ is assigned 595 to a server (local or remote). The evaluation is always done by the first element of the sorted set $F$, that is, the best-evaluated server at the moment. In lines 7-10, if the first element is the client itself, the Function AddTasksToClient is called (Algorithm 3). Otherwise, the Function AddTasksToServer is called (Algorithm 4). 
Finally, in lines 11-16, if there are no tasks left in $T$, the client processes tasks in $W$ locally, and the other tasks are sent to be executed in servers in $S$. If tasks remain, a problem has occurred, and the initial set of tasks $(Y)$ is all executed locally.

Function AddFeasibleServers. This Function checks each reply message $m$ received from a possible server $j$. This check is made to know if $j$ is feasible to execute tasks for the client. In line 3 of the Algorithm 2, $t_{j}$ receives the estimated time to execute the task $\tau \in T$ on the possible server $j$. This estimate, according to Sections 3.2 .3 and 3.3 .2 , considers several contextual parameters such as bandwidth, computational capacity, queue time, and others. In line 4, a check is made if $t_{j}$ is less than the estimated link lifetime between $i$ and $j$ (partial constraint C2 of problem P1). This estimated link lifetime is calculated according to Section 3.2.1. This calculation considers the communication ranges of the devices (WAVE or 5G) and that edge servers are static. Then, if the check of line 4 returns true, $F$ receives a tuple of the possible server $j$. This tuple contains, among other things, how much it will process $\left(h_{j}\right)$, how much data it will receive and return $\left(a_{z}\right)$, and the set of tasks that will process $\left(X_{j}\right)$, all initialized as zero or empty.

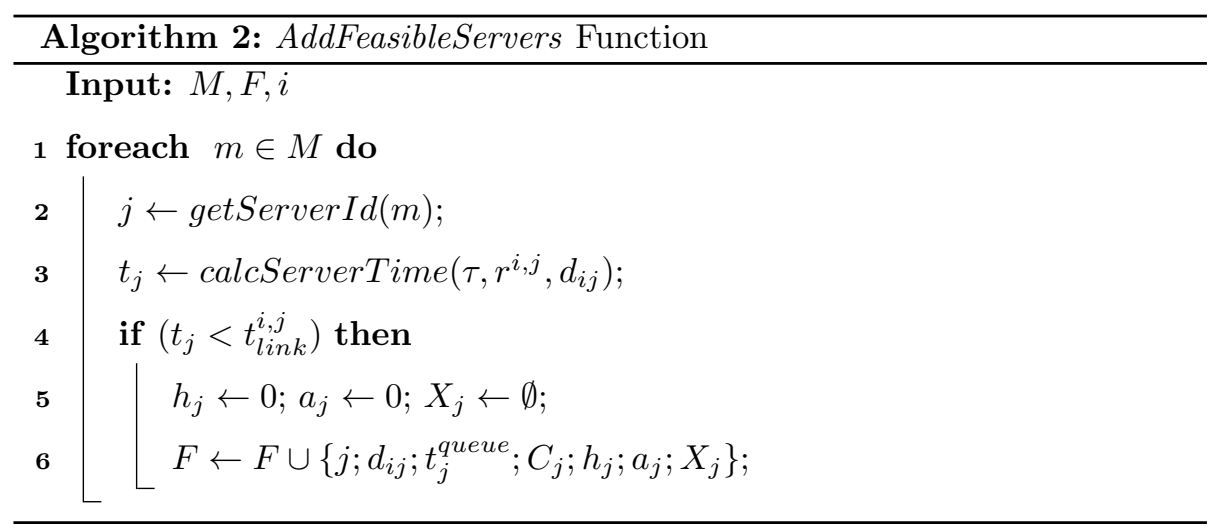

Function AddTasksToClient. It is responsible for assigning tasks to the client. In Algorithm 3, $W$ receives $\tau$, that is removed from $T$. Then, the 

in $i$ and considering the queue time and computational capacity. Finally, $F$ is sorted again.

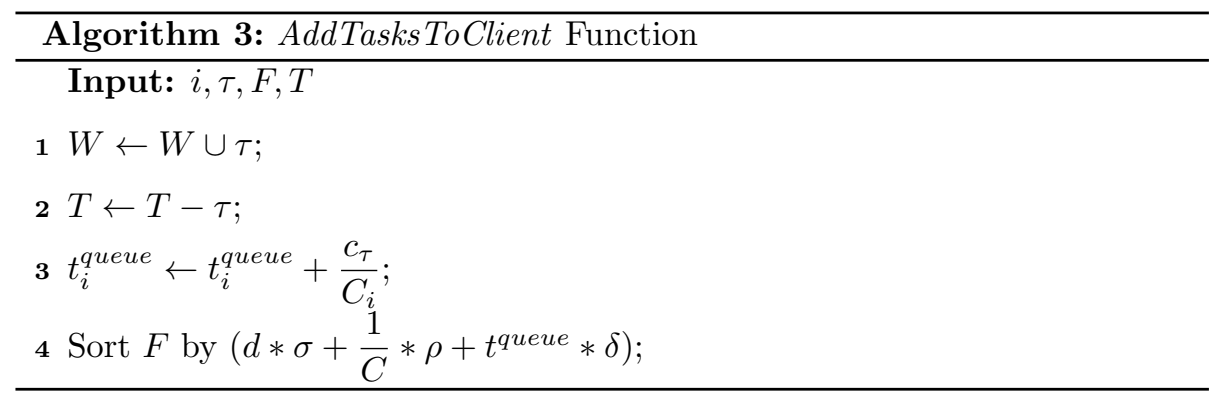
servers. In Algorithm 4 , line 1, as the server $z$ will process the task $\tau$, how much

625 will be received and returned $\left(a_{z}\right)$ receives an addition of $s_{\tau}^{u p}$ and $s_{\tau}^{\text {down }}$. In line $2, t_{z}$ receives the estimated time for server $z$ to execute its tasks. According to Sections 3.2 .3 and 3.3 .2 , this estimate considers several contextual parameters such as bandwidth, computational capacity, queue time, in addition to $h_{z}$ and 630 $a_{z}$. In lines $3-4$, if the route of the server is known $\left(K_{z}\right), l$ receives true if $i$ and $z$ are still within the communication range of each other after $t_{z}$ seconds. This last check is done through Function withinRange, following the calculations in Section 3.2 .2 Function withinRange assumes that $K_{z}$ is always true if $z$ is an edge server because it is static. In addition, the values of the WAVE and $5 \mathrm{G}$ ranges are also taken into account.

In lines 5-15, the algorithm checks whether the server can execute its assigned tasks (constraint $\mathrm{C} 2$ of the problem $\mathrm{P} 1$ ). With the estimate of the execution time of the assigned tasks, the verification is done by predicting positioning, if the route is known, or by the estimated link lifetime (considering the WAVE and $5 \mathrm{G}$ ranges of the devices and the immobility of edge servers). Suppose the check returns true (lines 5-11). Then, if the server is not in $S$, it is added. Next, $X_{z}$ receives $\tau, \tau$ is removed from $T$, the task queue of the server is increased 


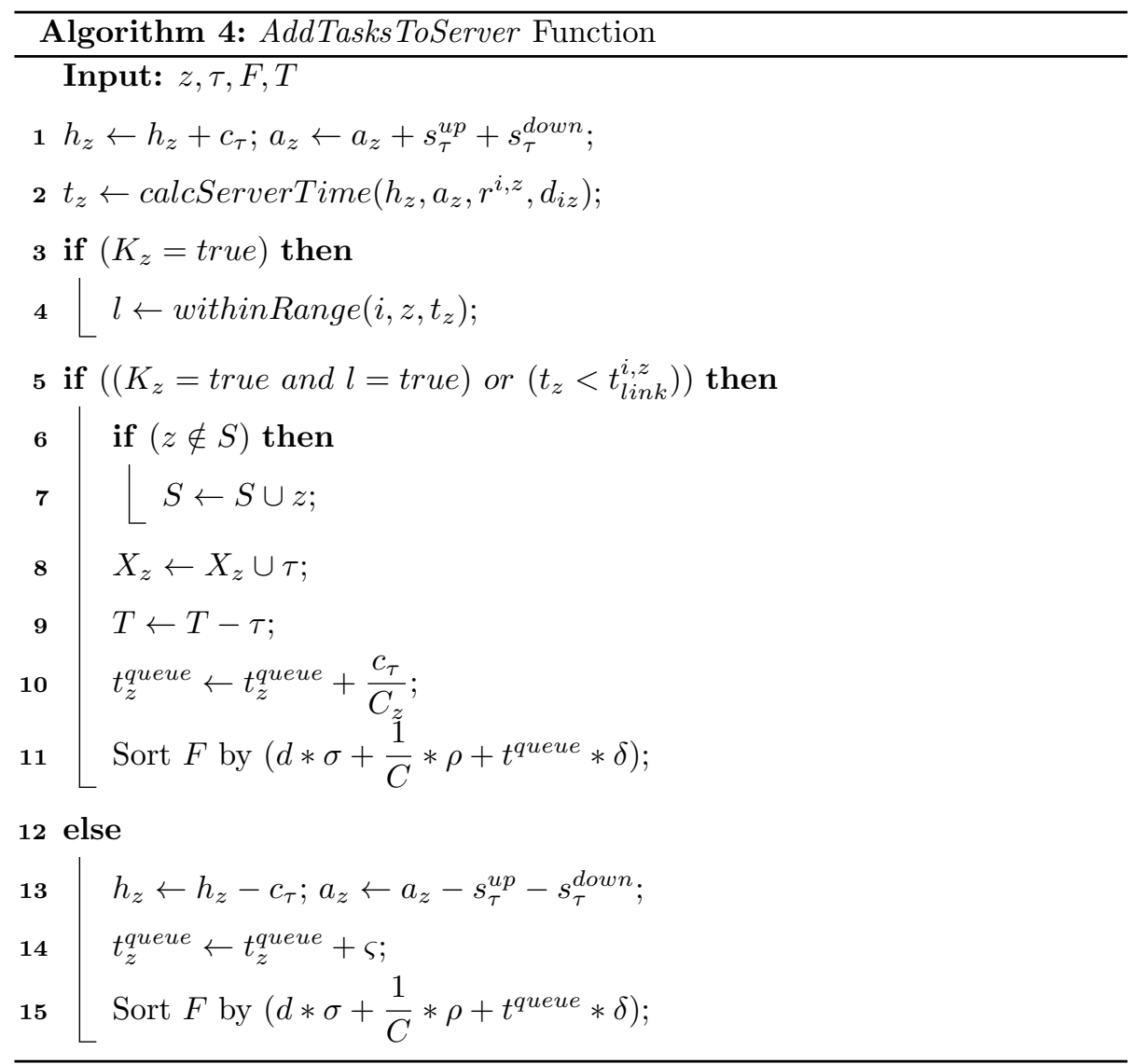

by the time to process $\tau$ (as if the task $\tau$ was already queued at server), and $F$ is sorted again. Suppose the check returns false (lines 12-15). In that case, the procedures for executing $\tau$ are undone, the queue of the server receives a high value constant $\varsigma$ so that the server stays in the last positions of $F$, and $F$ is sorted again.

Complexity Analysis. The most critical computational complexity aspect of the GTT algorithm is the loop of lines 5-10, which depends on the size of $T$. In each iteration of the loop, the algorithm executes the Function AddTasksToClient or the Function AddTasksToClient. Each of these functions has computational complexity determined mainly by the sorting of the set of feasible servers $F$, which is $O(F \log F)$ on the size of the set $F$. Since these sortings are 
done at each iteration of the loop of lines 5 -10, the computational complexity of the GTT algorithm is $O(T F \log F)$.

\subsubsection{Send/Receive Tasks}

Later the decision, the framework puts into practice the solution proposed by the GTT algorithm. Thus, the Decision Maker module transfers the tasks to the Distributor module that forwards the tasks to the appropriate modules (for local or remote execution). If the option is to send some tasks to remote devices, they are sent to the WAVE or $5 G$ modules. In this way, as shown in Figure 4, tasks are distributed to remote servers (vehicle and edge server) to start processing. Thus, multiple servers can simultaneously collaborate to provide computing services to the client.

Figure 4: Client (red) sends tasks to the chosen servers. Two tasks are sent to be executed on the edge server (whose base station is within its $5 \mathrm{G}$ communication range - light blue background) via V2I connection (purple dotted line). One task is sent to be executed on the vehicle ahead (within its WAVE communication range - black dotted line) via V2V connection (blue dotted line).

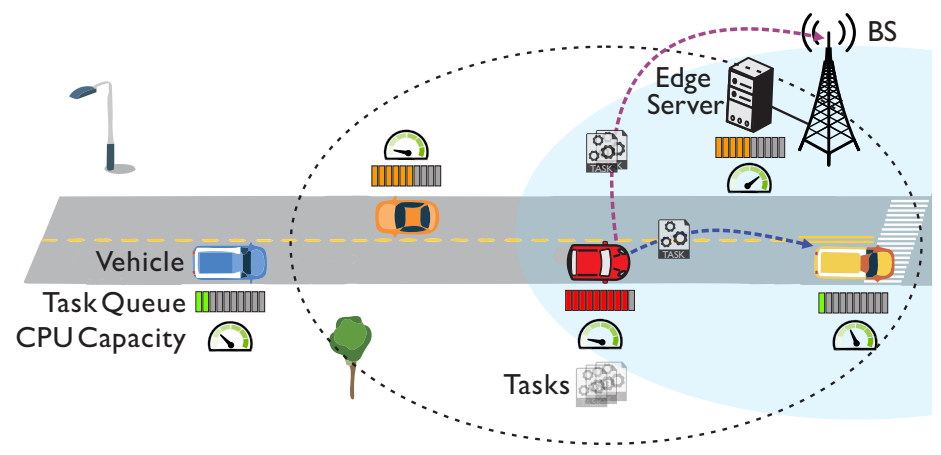

Following processing, each chosen server returns the processing result to the client. This result comes through the WAVE or $5 G$ modules. Then, the result passes through the Data and Context Gatherer module and finally reaches the Application to continue its execution. 


\subsubsection{Failure recovery}

After the client sends the tasks to the remote servers, it triggers a function to monitor the connectivity between client and servers, through the Data and Context Gatherer module. If beacon messages from a server continue to be received by the client, connectivity still exists. If no beacon messages arrive from a server in $\xi$ milliseconds, and if the processing result from that server has not yet been returned, a failure is detected. In this case, the Data and Context Gatherer module informs the Decision Maker module. The Decision Maker module activates the Task Distributor module that has the tasks of the lost server and distributes it to the Task Queue to be executed locally on the client.

\section{Experiments}

This section presents the details of the experiments performed to evaluate the proposed framework and algorithm. All experiments follow the general network structure, communication, and computation models described in Section 3. Table 4 presents a summary of the main characteristics of the experiments. comparison with other algorithms are detailed below.

\subsection{Network}

We employ simulation-based experiments to evaluate the proposed framework. For this, we used the ns-3 simulator [49] (version 3.29) running on a computer with an Intel Xeon E5645 processor @ 2.40GHz and 32 GB RAM. All scenarios use common packet traffic on vehicular networks.

\subsection{Scenario}

To build the simulated scenarios, we use Simulation of Urban MObility (SUMO) [50. The first scenario, seen in Figure 5, consists of a highway in

${ }_{695}$ a metropolitan area with the following characteristics: $5 \mathrm{~km}$ long, two lanes in each direction, and a maximum speed of $60 \mathrm{~km} / \mathrm{h}$. 
Table 4: Main simulation parameters.

\begin{tabular}{|ll|}
\hline General & \\
Scenarios & Highway and Urban \\
\# of vehicles in highway & 11,55, and 120 per km \\
Simulation time & 25,120, and 250 per km ${ }^{2}$ \\
\# of simulations carried out & 200 times \\
Mobility model & Krauss \\
Servers offering offloading & All (vehicles and edge servers) \\
Known Routes of Vehicles & $50 \%$ \\
CPU Capacity of Edge Servers & $1.5,2.0$, and $2.5 \mathrm{GHz}$ \\
CPU Capacity of Vehicles & 0.5 and $1.0 \mathrm{GHz}$ \\
CPU Requirement of a Task & 3.5 Gigacycles \\
Task size (upload) & 558 KB \\
Result size (download) & 1000 B \\
Workload type & ALPR \\
Workloads & $4,6,8,10$, and 12 tasks \\
Transport protocol & UDP (discovery), TCP (offloading) \\
Others packets traffics & Beacon messages \\
WAVE & \\
Communication range & 250 meters \\
Radio propagation model & Two-Ray Ground \\
Layer 2 protocol & 27 Mbps \\
Data rate & \\
Communication range & \\
\hline
\end{tabular}

The second scenario, seen in Figure 6 , consists of an urban area with $2 \mathrm{~km}^{2}$ area and a maximum speed of $60 \mathrm{~km} / \mathrm{h}$. 
Figure 5: Highway scenario used in the experiments. An adapted stretch of a Brazilian highway with returns at the ends. The black dotted lines highlight the smaller section showing vehicles and a $5 \mathrm{G}$ base station with a coupled edge server.
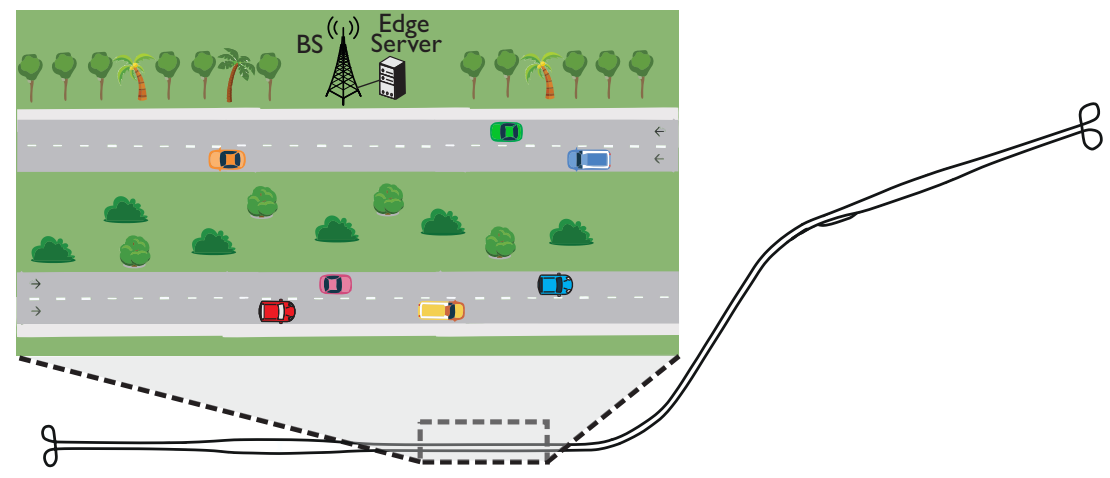

Figure 6: Urban scenario used in the experiments. An adapted stretch of the Manhattan region, New York, USA. The black dotted lines highlight an intersection showing vehicles and a $5 \mathrm{G}$ base station with a coupled edge server.

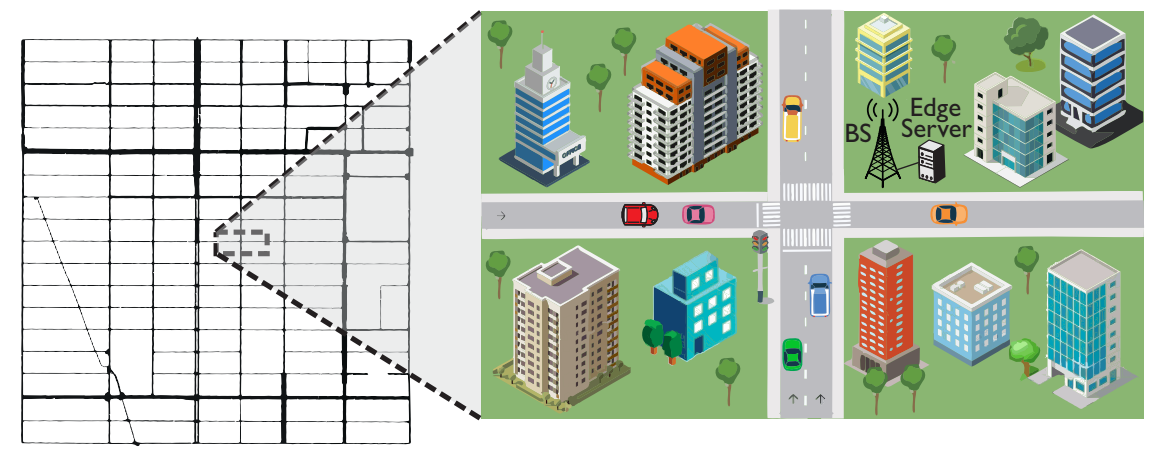

\subsection{Mobility}

We consider three types of nodes with predefined quantity: vehicles, edge servers, and base stations. Base stations and edge servers have a fixed location and are spatially distributed to provide complete communication coverage.

Concerning vehicles, their initial positions are spatially and randomly distributed across different points in the scenario. They follow random paths with different starting and ending points. The vehicles move at different speeds and directions, according to the microscopic car-following model of Krauss. Thus, 
the vehicle's speed depends on the maximum speed of the road, the speed of the vehicle ahead (if any and not to collide), the difference in vehicle positions, and static parameters such as the driver's reaction time [50, 51].

The simulator used to generate the mobility of the network nodes was the SUMO. It was chosen for having mobility models that reflect the real-world behavior of vehicular traffic and integration mechanisms with network simulators. Thus, most computation offloading experiments in vehicular scenarios utilize simulation-generated mobility, with SUMO being one of the most used simulators [9, 50].

\subsection{Vehicular Density}

For each scenario, we use three types of vehicular density: low, medium, and high. Vehicular density is considered low if it has approximately 11 vehicles $/ \mathrm{km}$ in highway scenario and 25 vehicles $/ \mathrm{km}^{2}$ in urban scenario. In medium density are there are approximately 55 vehicles/km in highway scenario and 120 vehicles $/ \mathrm{km}^{2}$ in urban scenario. Finally, in high density, we have approximately 120 vehicles $/ \mathrm{km}$ in highway scenario and 250 vehicles $/ \mathrm{km}^{2}$ in urban scenario 9.

\subsection{Application}

The application used was Automatic License Plate Recognition (ALPR), which consists of image capture, vehicle detection, plate detection, and optical character recognition. For each captured image, we used SSD-300 with MobileNet [52] to detect vehicles and Tiny YOLOv3 [53] to identify the license plate. Then, we used an algorithm of optical character recognition to recognize the characters. As this application's tasks are compute-intensive, the vehicles can offload them to be executed on remote servers (edge servers or other vehicles) to reduce the execution time.

In our experiments, we considered an ALPR task $(\tau)$ involving independent images with a total number of necessary CPU cycles equal to 3.5 Gigacycles ${ }_{735}\left(c_{\tau}=3.5 G c\right)$, size of the upload image equal to $558 \mathrm{~KB}\left(s_{\tau}^{u p}=558 \mathrm{~KB}\right)$ and 
the size of the processing results equal to $1000 \mathrm{~B}\left(s_{\tau}^{\text {down }}=1000 \mathrm{~B}\right.$, representing strings containing each recognized license plate). We also consider five different types of ALPR workloads. Workloads 1, 2, 3, 4 and 5 have, respectively, 4, 6, 8, 10 and 12 tasks of type $\tau$.

As it is not possible to simulate the workload processing in ns-3, we use real offloading experiments. In this way, we calculate the execution times of ALPR tasks and the size of the packets transferred during the offloading process. For edge servers, we use CPUs with capacities of 1.5, 2.0, and 2.5 GHz. For vehicles, we use CPUs with 0.5 and $1.0 \mathrm{GHz}$.

\subsection{Comparison with other Algorithms}

We compared the proposed decision algorithm (GTT) with four other algorithms: FIFO (First In, First Out), HVC (Hybrid Vehicular edge Cloud) 32, MDO (Multi-Decision based Offloading) [19, and GCF (Greedy for CPU Free) 33. All algorithms used the failure recovery mechanism described in Section

7504.2 .4 and were tested under the same scenarios and conditions. The FIFO algorithm was implemented to select the servers that reply first, sending a task to each one. If the FIFO does not find enough servers, it executes all tasks locally. The HVC, MDO, and GCF were implemented following the descriptions of their respective papers. The HVC algorithm prioritizes sending the requests via $5 \mathrm{G}$ (in our scenario, to the edge server). The MDO uses only neighboring vehicles as remote servers, choosing the capable ones and with the best scores (based on the relative speeds between vehicles and waiting times). The GCF algorithm seeks servers close to the client and with the highest free CPU availability (low queue times) in the sequence: edge servers, client, nearby vehicles. However, the GCF does not take into account the computational capacity of the network nodes. In addition, known routes of vehicles are not considered in FIFO, HVC, $\mathrm{MDO}$, and GCF. 


\section{Results Analysis}

This section discusses the main results obtained from the experiments men-

765

\subsection{Impact of Known Routes of Vehicles on the Number of Recovered Tasks}

As mentioned earlier, some vehicles can share their routes with other nodes in the network. To analyze the impact of this information shared in computation offloading, we used the urban scenario for having more different route options. Besides, we used the workload with 12 tasks and the algorithm that uses information about known routes (i.e., GTT). Such a number of tasks was chosen because distributing more tasks increases the chance of failure.

In Figure 7, we can see that the more vehicles with known routes (x-axis), the greater the reduction of failures/recovered tasks. For example, in low vehicular by $15.1 \%$ and $50.0 \%$, respectively, the number of tasks recovered. In medium density, the reduction was from $31.7 \%$ and $48.8 \%$ to $50 \%$ and $100 \%$ of vehicles with known routes, respectively. With high vehicular density, having 50\% and $100 \%$ of vehicles with known routes helped GTT to reduce the number of tasks

\subsection{Type of Occurrence per Task}

This section presents the performance of the proposed algorithm and others from the literature concerning what happens with each task of the different workloads. Thus, in Figures 8 and 9, a task can have three types of occurrences: TL, TS, or TR. TL (parts of the bars with checkered lines) represents tasks executed locally by the client. TS (part of the bars without lines) represents tasks that were successfully offloaded, executed remotely, and the results were returned to the client. TR (parts of the bars with simple diagonal lines) represents tasks that were offloaded, and there was some failure in the process, causing them to need recovery on the client, as seen in Section 4.2.4. 
Figure 7: Impact of known routes of vehicles on the number of failures/recovered tasks with low, medium, and high vehicular density. The baseline is the number of recovered tasks when the percentage of known routes is zero. Scenario: urban, 12 tasks, and GTT algorithm.

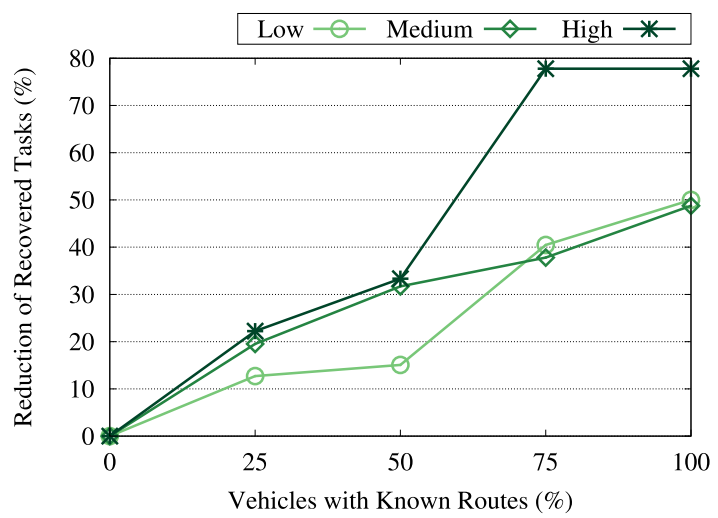

In Figures $8 \mathrm{a}$ to $8 \mathrm{c}$, we have the percentage of tasks by type of occurrence of the FIFO, HVC, MDO, GCF, and GTT algorithms in the highway scenarios. We can see that the GTT has the highest percentages of TS and low percentages of TR. With low vehicular density (Figure 8a), the percentage of TS in GTT was on average $75.0 \%$, while in FIFO, HVC, MDO, and GCF, it was, respectively, $34.4 \%, 63.6 \%, 53.3 \%$, and $71.9 \%$. The average percentage of TR in GTT was 1.6\%, while in FIFO, HVC, MDO, and GCF were, respectively, 9.4\%, 16.8\%, $1.5 \%$, and $5.8 \%$. In the medium density scenario (Figure $8 \mathrm{~b}$ ), the percentage of TS in GTT was on average 88.6\%, while in FIFO, HVC, MDO, and GCF it was, respectively, $63.3 \%, 73.4 \%, 71.0 \%$, and $87.1 \%$. Regarding the average percentage of TR, that of GTT was $1.4 \%$, while FIFO, HVC, MDO, and GCF were, respectively, $17.7 \%, 15.6 \%, 0.3 \%$, and $4.7 \%$. With high vehicular density (Figure 8c), the GTT had a higher average percentage of TS (89.8\%) than FIFO $(62.3 \%)$, HVC (74.9\%), MDO (66.0\%), and GCF (88.2\%). Finally, the average percentage of TR in GTT was 1.4\%, while in FIFO, HVC, MDO, and GCF, it was $19.1 \%, 14.0 \%, 0.3 \%$, and $4.7 \%$, respectively.

In Figures $9 \mathrm{a}$ to $9 \mathrm{c}$, we present the percentage of tasks by type of occurrence of the FIFO, HVC, MDO, GCF, and GTT algorithms in the urban scenarios. 


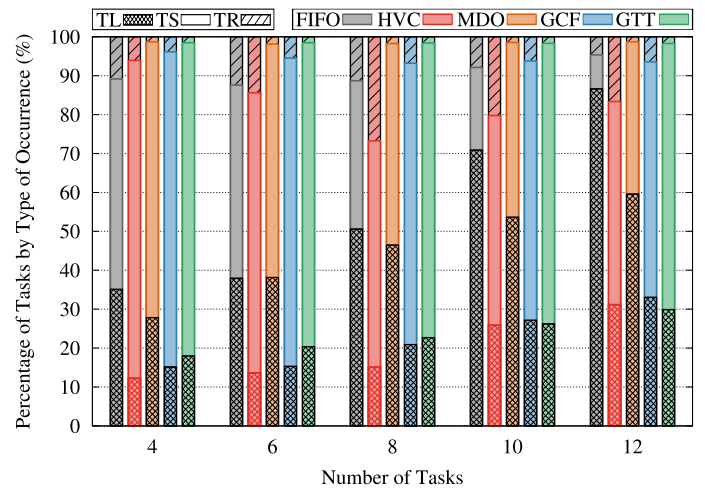

(a) Low vehicular density.

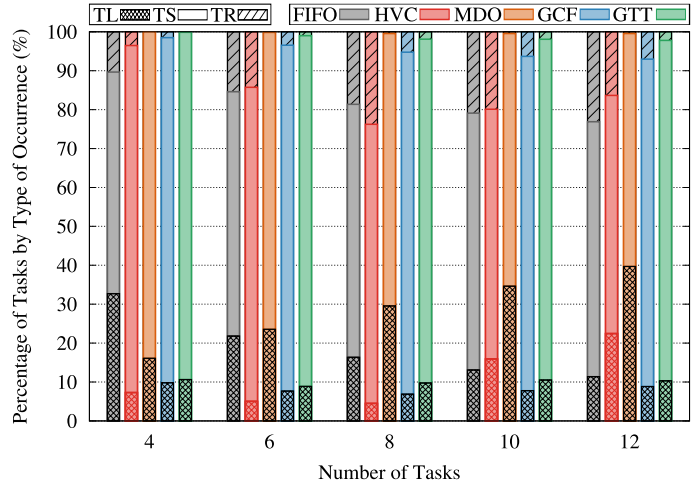

(b) Medium vehicular density.

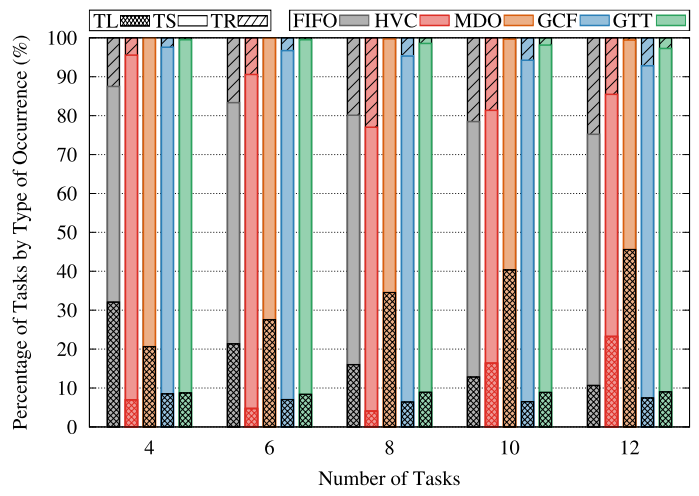

(c) High vehicular density.

Figure 8: Percentage of tasks by type of occurrence for workloads with 4, 6, 8, 10, and 12 tasks. We can have three types of occurrences: TL - task executed locally; TS - task successfully executed remotely; TR - task executed with recovery. For comparison, we used five algorithms: FIFO, HVC, MDO, GCF, and GTT in highway scenarios. 
We can also see that GTT has a higher percentage of TS and a lower percentage of TR than other algorithms. In low density scenario (Figure 9a), GTT had, on average, a percentage of TS of $67.6 \%$, while FIFO, HVC, MDO, and GCF had, respectively, $22.9 \%, 66.1 \%, 52.5 \%$, and $61.4 \%$. In terms of TR, the GTT's average percentage was $3.2 \%$, the FIFO was $5.2 \%$, the $\mathrm{HVC}$ was $4.1 \%$, the MDO was $3.6 \%$, and the GCF was $3.6 \%$. With medium vehicular density (Figure 9b), the average percentage of TS in GTT was $83.8 \%$, while in FIFO, HVC, MDO, and GCF it was, respectively, $60.8 \%, 77.0 \%, 75.3 \%$, and $79.2 \%$. The average percentage of TR in GTT was 1.0\%, while in FIFO, HVC, MDO, and GCF, it was, respectively, $9.0 \%, 4.0 \%, 2.3 \%$, and $3.7 \%$. With high vehicular density (Figure 9c), GTT had an average TS percentage of $88.6 \%$, while FIFO, HVC,

$820 \mathrm{MDO}$, and GCF had, respectively, $66.6 \%, 80.3 \%, 81.0 \%$, and $84.7 \%$. Finally, on average, the percentage of TR for GTT was $0.8 \%$, while for FIFO, HVC, MDO, and GCF, it was, respectively, $11.5 \%, 4.5 \%, 1.8 \%$, and $3.5 \%$.

\subsection{Reduction in Execution Time}

This section compares the performance of each algorithm concerning the metric of reduction in execution time of different workloads. This metric is calculated by comparing each algorithm's time with the baseline, which is the time to execute all tasks locally on the client ${ }^{1}$. The error bars in the figures of this section show the $95 \%$ confidence interval of the corresponding data based on the standard normal distribution. Furthermore, the time taken to decide where each task should be processed was around 10 nanoseconds for all algorithms considered.

Thus, in the highway scenarios (Figures 10a to 10c), we can see that the most significant reductions in execution time, in general, are from the GTT algorithm. For example, in the low vehicular density scenario (Figure 10a), 835 on average, GTT algorithm had a reduction in execution time of $59.4 \%$. In contrast, FIFO, HVC, MDO, and GCF algorithms had, respectively, reductions

\footnotetext{
${ }^{1} \mathrm{~A}$ similar comparison was made with this same baseline in 32 .
} 


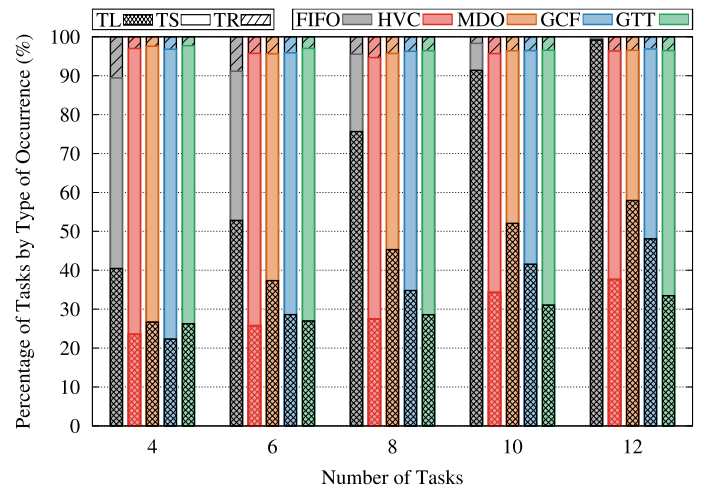

(a) Low vehicular density.

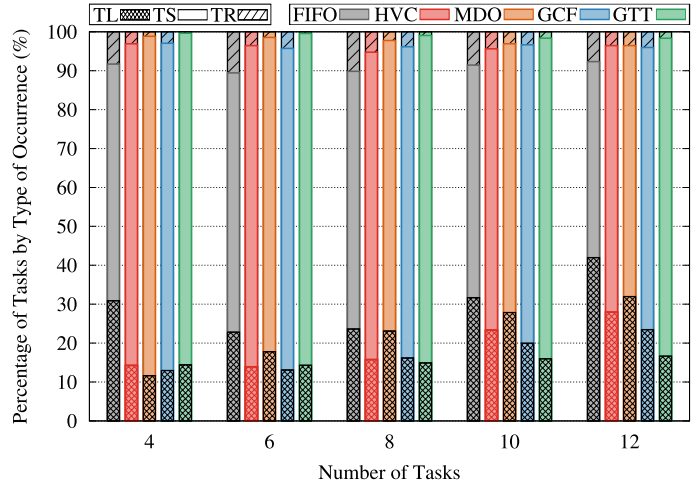

(b) Medium vehicular density.

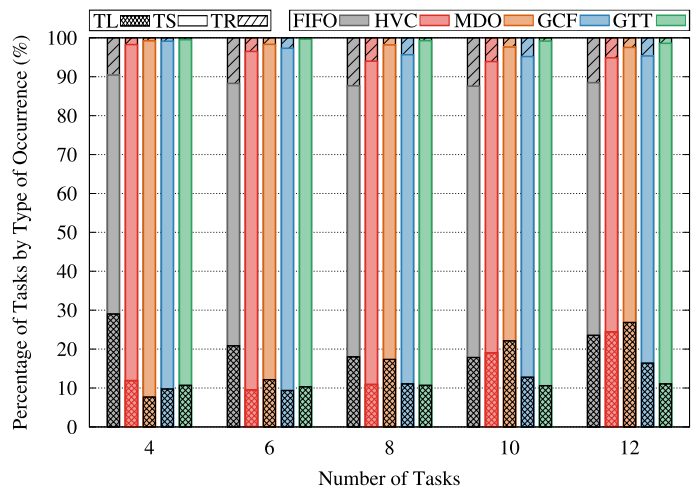

(c) High vehicular density.

Figure 9: Percentage of tasks by type of occurrence for workloads with 4, 6, 8, 10, and 12 tasks. We can have three types of occurrences: TL - task executed locally; TS - task successfully executed remotely; TR - task executed with recovery. For comparison, we used five algorithms: FIFO, HVC, MDO, GCF, and GTT in urban scenarios. 
of $18.2 \%, 33.6 \%, 35.3 \%$, and $52.1 \%$. In medium density (Figure 10b), GTT had an average reduction of $69.7 \%$, while FIFO, HVC, MDO, and GCF had, respectively, reductions of $37.8 \%, 44.2 \%, 50.8 \%$, and $62.9 \%$. With high vehicular density (Figure 10c), GTT had an average reduction of $70.3 \%$, while FIFO, HVC, MDO, and GCF had, respectively, reductions of $36.3 \%, 45.4 \%, 49.2 \%$, and $64.0 \%$.

In urban scenarios (Figures 11a to 11c), we can see that the greatest reductions in execution time are also, in general, of the GTT algorithm. For example, in the low vehicular density scenario (Figure 11a), on average, GTT algorithm had a $52.4 \%$ reduction in execution time. On the other hand, FIFO, HVC, MDO, and GCF algorithms had, respectively, reductions of $9.5 \%, 38.8 \%, 32.1 \%$, and 42.7\%. In the medium density scenario (Figure 11b), the average reduction in GTT was $64.8 \%$, while in FIFO, HVC, MDO, and GCF, it was, respectively, ${ }_{850} 35.3 \%, 49.6 \%, 48.2 \%$, and 53.8\%. With high vehicular density (Figure 11c), GTT had an average reduction of $68.9 \%$, while FIFO, HVC, MDO, and GCF had, respectively, average reductions of $40.3 \%, 53.3 \%, 55.9 \%$, and $59.2 \%$.

\subsubsection{Statistical Tests}

As shown in Figures 10 and 11. GTT has the most significant reductions in task execution time. However, in Figures 10a and 11a, there are overlapping confidence intervals between the GTT and the GCF with four and six tasks. This type of overlapping also exists in Figure 11a between GTT and HVC with four tasks. Then, we performed two-sample t-tests (one-tailed) to assess pairwise whether there are significant differences in the performance of the algorithms in these cases. The null hypothesis $(H 0)$ is that the averages of the two algorithms evaluated in each case are equal. However, in all tests, we obtained p-values less than 0.05 , rejecting $H 0$. Therefore, the GTT average reductions in execution time are significantly different from the other algorithms in all evaluated cases. 


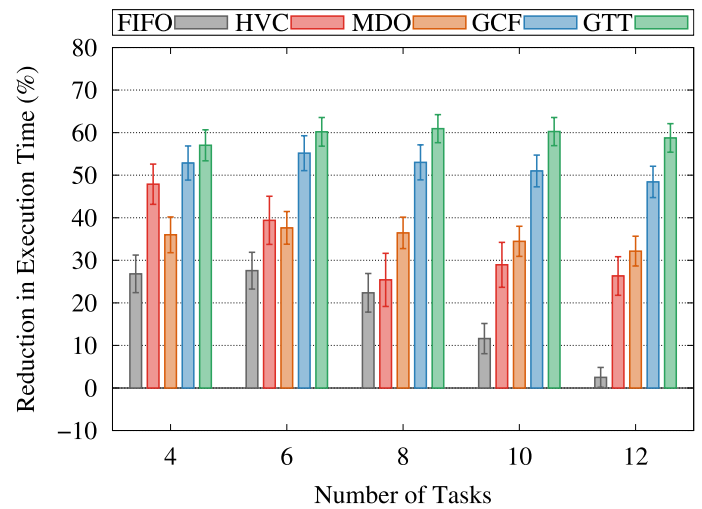

(a) Low vehicular density.

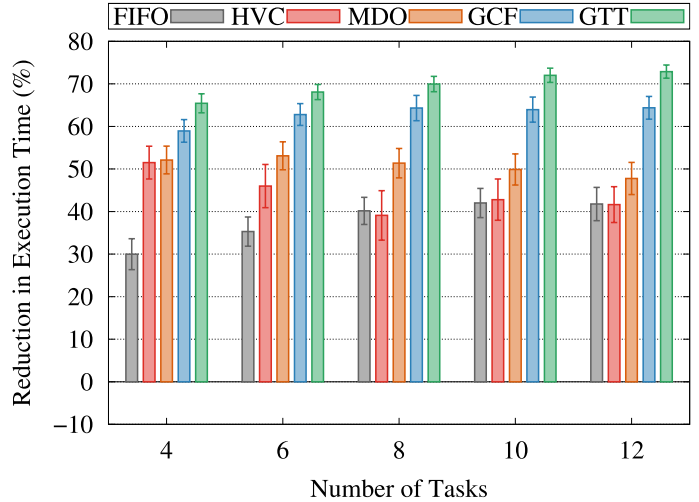

(b) Medium vehicular density.

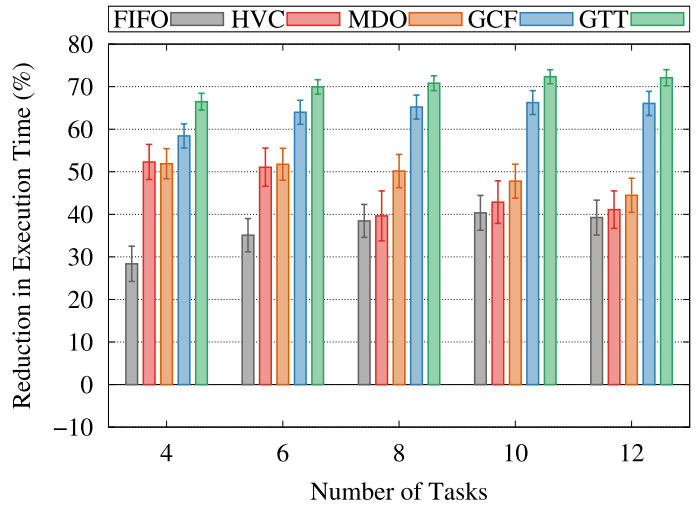

(c) High vehicular density.

Figure 10: Average reduction in execution time, with $95 \%$ confidence interval, for workloads with $4,6,8,10$, and 12 tasks. The baseline is the execution time when the workload is fully executed on the client. For comparison, we used five algorithms: FIFO, HVC, MDO, GCF, and GTT in highway scenarios. 


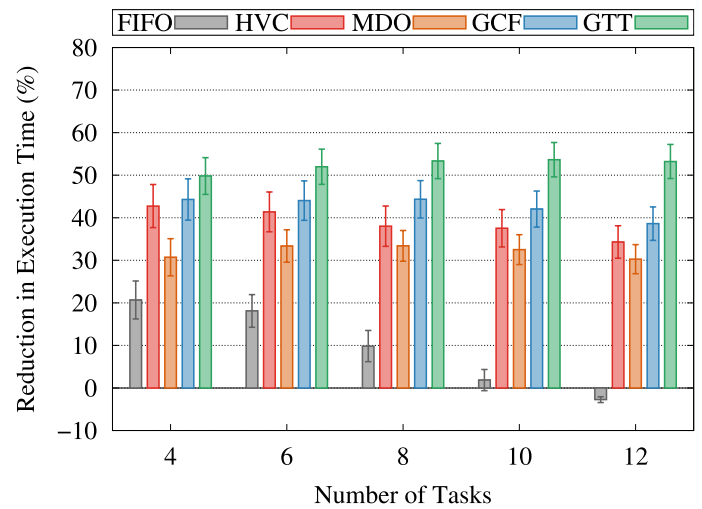

(a) Low vehicular density.

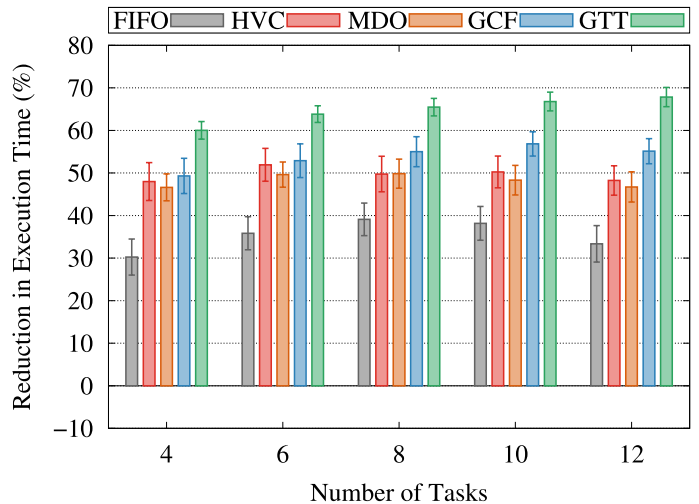

(b) Medium vehicular density.

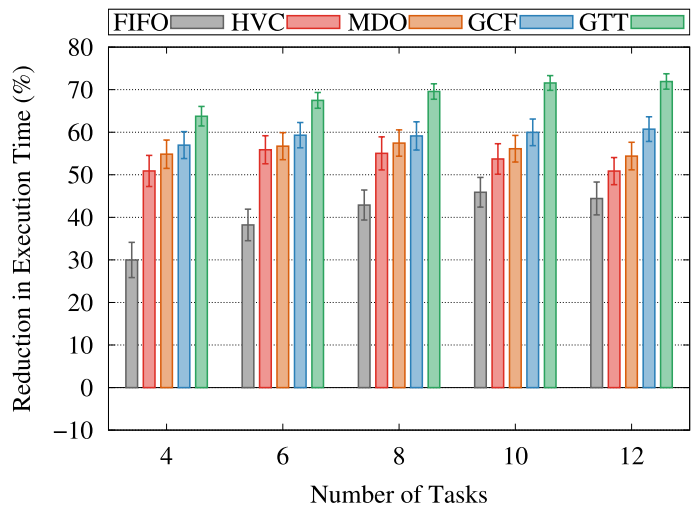

(c) High vehicular density.

Figure 11: Average reduction in execution time, with 95\% confidence interval, for workloads with $4,6,8,10$, and 12 tasks. The baseline is the execution time when the workload is fully executed on the client. For comparison, we used five algorithms: FIFO, HVC, MDO, GCF, and GTT in urban scenarios. 


\subsection{Discussion} sities, the information of known routes of vehicles reduces the number of tasks recovered, i.e., the number of failures. This reduction occurs because this realtime contextual information helps GTT to better predict the network nodes' position at a given time. Consequently, GTT is able to calculate more precisely the best server to send the tasks so that failures/recoveries do not happen.

We also note in Figures 8 and 9 that the GTT has the highest percentages of tasks successfully executed remotely (TS) and the lowest percentages of tasks recovered (TR) in most cases. Having a high TS percentage is a good indicator because it relieves the client's processing overload and tasks can be executed more quickly on remote servers with better computing resources. A low TR percentage is also an important indicator. Sending tasks to be performed remotely involves using bandwidth and processing on the remote server. It also involves time to transmit, process, and wait for their results. When a failure occurs, these times and computational resources are wasted. Also, as the result time is still needed to detect the failure and re-execute the task locally. Thus, a low $\mathrm{TR}$ percentage indicates a low percentage of failures, saving time and computational resources.

In Figures 10 and 11, with the most significant reductions in execution time compared to other algorithms, GTT can help an application to execute its tasks more quickly. These reductions are very important in environments as dynamic as vehicular networks. In this sense, the GTT's best time performance is confirmed by Section 6.3.1. In it, we can see that GTT has execution times significantly different from all other algorithms, including GCF (algorithm with performance closer to GTT) in all scenarios. Another interesting point is that the algorithms spent negligible time deciding where tasks should be processed. Hence, this time did not influence the workloads' execution times.

Therefore, the results show that computation offloading processes can reduce an application's total execution time in vehicular systems. We have also seen 
that GTT has the greatest reductions in execution times of tasks, the highest rates of tasks successfully offloaded, and the lowest failure rates (with fewer recovered tasks in most cases) compared to other algorithms.

Some factors can explain the better performance of the GTT. First, as a greedy algorithm, GTT periodically updates the best possible server (including the client itself) to execute each task, taking advantage of the computational resources available from all nodes in the network. This evaluation of the best server is done through various real-time contextual information parameters. In this way, with more contextual information, evaluations become more accurate. Consequently, GTT is able to make better offloading decisions, choosing the best servers with feasible link lifetimes, shorter distances, and good CPU availability and capacity. With this, servers can execute tasks faster and the results can be returned to the client before they lose connectivity, avoiding failures.

Second, if available, information about known routes of vehicles contributes to reducing the offloading processes' failures and, consequently, reducing task recoveries. With fewer recoveries, the reduction in the total execution time of the workload is greater. The other algorithms, with more recoveries, end up delaying more their total execution time. In the case of the MDO, although it has low percentages of $\mathrm{TR}$, it does not take advantage of the computational resources of edge servers and has more tasks executed locally (TL), overloading client vehicles.

Third, the simultaneous use of the WAVE and 5G networks also contributes to GTT's good performance. This use allows the GTT to take advantage of both the vehicular clouds (with V2V) and the edge servers (with V2I). It also allows to increase the transmission capacity of tasks and reduce latencies. Besides, it combines the independence of infrastructure and direct communications from WAVE networks and the large data rates and increased band spectrum availability of $5 \mathrm{G}$ networks.

In this way, GTT makes more accurate contextual evaluations, better server choices, better task assignment, and takes advantage of technological advantages with the simultaneous use of WAVE, 5G, and the vehicular edge computing 
system. Through extensive simulations and diverse vehicular environments, we show that GTT achieves the greatest reductions in the total execution times of tasks and the lowest rates of offloading failures in most cases, providing good and feasible solutions to problem P1.

\section{Conclusion}

This work presented a context-oriented framework for computation offloading in vehicular edge computing systems with the objective of reliably improving the performance of the application. The framework modules support discovering computational resources, gathering real-time contextual data, distributing computation tasks, and providing failure recovery. Then, the core of the framework, a greedy algorithm called GTT, can focus on task assignment, deciding where each application task should be executed. For this decision, GTT taking contextual information into account and taking advantage of WAVE and $5 \mathrm{G}$ networks and the computing resources of vehicular clouds and edge servers.

940 Through extensive experiments in different vehicular environments and workloads, we evaluated the proposed framework's efficiency and the GTT algorithm compared to other solutions in the literature. As seen in the results, on average, GTT achieved to offload up to $89.4 \%$ of all tasks and needed to recover only $0.8 \%$ of them. Besides, on average, GTT has reduced up to $70.3 \%$ of tasks execution time compared to entirely local execution and up to $42.9 \%$ if compared to other algorithms. Thus, our solution showed good adaptation to the challenges encountered, such as fast vehicular mobility, contextual information gathering, and NP-hardness of the task distribution decision. Our solution also performed better than other solutions in terms of reducing total execution time and failure rates. Some characteristics of the GTT contributed to obtaining the best performance. For example, the use of diverse real-time contextual information parameters contributed to finding the best possible servers for each task of the application, executing the tasks more quickly, and minimizing failures. The special information about known routes of vehicles made it possible to pre- 

this information can reduce the number of failures/recoveries by up to $77.8 \%$. Finally, the WAVE and 5G networks' simultaneous use increased the task transmission capacities, decreasing communication delays and remote task execution time. In future works, we intend to improve our solution by considering the processes

\section{References}

[1] K. Wevers, M. Lu, V2x communication for its-from ieee $802.11 \mathrm{p}$ towards 5g, IEEE 5G Tech Focus 1 (2) (2017) 5-10.

965

[4] M. Mezzavilla, M. Zhang, M. Polese, R. Ford, S. Dutta, S. Rangan, M. Zorzi, End-to-end simulation of $5 \mathrm{~g}$ mmwave networks, IEEE Commun. Surv. Tutor. 20 (3) (2018) 2237-2263.

[5] C. R. Storck, F. Duarte-Figueiredo, A 5g v2x ecosystem providing internet

[6] J. Zhang, K. B. Letaief, Mobile edge intelligence and computing for the internet of vehicles, Proc. IEEE 108 (2) (2019) 246-261.

[7] A. Boukerche, V. Soto, Computation offloading and retrieval for vehicular edge computing: Algorithms, models, and classification, ACM Comput.

[3] D. Jiang, L. Delgrossi, Ieee 802.11 p: Towards an international standard for wireless access in vehicular environments, in: Vehicular Technology Conference, 2008. VTC Spring 2008. IEEE, IEEE, 2008, pp. 2036-2040. of vehicles, Sens. 19 (3) (2019) 550.

Surv. (CSUR) 53 (4) (2020) 1-35. 
[8] Y. Liu, S. Wang, Q. Zhao, S. Du, A. Zhou, X. Ma, F. Yang, Dependencyaware task scheduling in vehicular edge computing, IEEE Internet Things J. 7 (6) (2020) 4961-4971. doi:10.1109/JIOT.2020.2972041.

[9] A. B. Souza, P. A. Rego, T. Carneiro, J. C. Rodrigues, P. P. Rebouças Filho, J. N. Souza, V. Chamola, V. H. C. Albuquerque, B. Sikdar, Computation offloading for vehicular environments: A survey, IEEE Access 8 (2020) 198214-198243. doi:10.1109/ACCESS.2020.3033828.

[10] P. A. Rego, P. B. Costa, E. F. Coutinho, L. S. Rocha, F. A. Trinta, J. N. de Souza, Performing computation offloading on multiple platforms, Comput. Commun. 105 (2017) 1-13.

[11] D. Xu, Y. Li, X. Chen, J. Li, P. Hui, S. Chen, J. Crowcroft, A survey of opportunistic offloading, IEEE Commun. Surv. Tutor. 20 (3) (2018) 21982236.

[12] H. Vahdat-Nejad, A. Ramazani, T. Mohammadi, W. Mansoor, A survey on context-aware vehicular network applications, Veh. Commun. 3 (2016) $43-57$.

[13] Y. Shin, H. Choi, Y. Nam, E. Lee, Data delivery protocol using the trajectory information on a road map in vanets, Ad Hoc Netw. 107 (2020) 102260.

[14] S. Wang, C. Ding, N. Zhang, X. Liu, A. Zhou, J. Cao, X. S. Shen, A cloud-guided feature extraction approach for image retrieval in mobile edge computing, IEEE Trans. on Mob. Comput.

[15] M. Tang, L. Gao, J. Huang, Enabling edge cooperation in tactile internet via 3c resource sharing, IEEE J. on Sel. Areas in Commun. 36 (11) (2018) $2444-2454$.

[16] Y. Li, X. Wang, X. Gan, H. Jin, L. Fu, X. Wang, Learning-aided computation offloading for trusted collaborative mobile edge computing, IEEE Trans. on Mob. Comput. 19 (12) (2019) 2833-2849. 
[17] L. Liu, C. Chen, Q. Pei, S. Maharjan, Y. Zhang, Vehicular edge computing and networking: A survey, Mob. Netw. and Appl. (2020) 1-24.

[18] G. Fan, H. Jin, Q. Liu, W. Qin, X. Gan, H. Long, L. Fu, X. Wang, Joint scheduling and incentive mechanism for spatio-temporal vehicular crowd sensing, IEEE Trans. on Mob. Comput.

[19] A. U. Rahman, A. W. Malik, V. Sati, A. Chopra, S. D. Ravana, Contextaware opportunistic computing in vehicle-to-vehicle networks, Veh. Commun. 24 (2020) 100236.

[20] M. Liwang, J. Wang, Z. Gao, X. Du, M. Guizani, Game theory based opportunistic computation offloading in cloud-enabled iov, IEEE Access 7 (2019) 32551-32561.

[21] M. Charitos, G. Kalivas, Mimo hetnet ieee 802.11 p-lte deployment in a vehicular urban environment, Veh. Commun. 9 (2017) 222-232.

[22] N. Dreyer, A. Moller, Z. H. Mir, F. Filali, T. Kurner, A data traffic steering algorithm for ieee $802.11 \mathrm{p} /$ lte hybrid vehicular networks, in: 2016 IEEE 84th Vehicular Technology Conference (VTC-Fall), IEEE, 2016, pp. 1-6.

[23] S. Ucar, S. C. Ergen, O. Ozkasap, Multihop-cluster-based ieee 802.11 p and lte hybrid architecture for vanet safety message dissemination, IEEE Trans. Veh. Technol. 65 (4) (2015) 2621-2636.

[24] Z. Ning, X. Wang, J. J. Rodrigues, F. Xia, Joint computation offloading, power allocation, and channel assignment for $5 \mathrm{~g}$-enabled traffic management systems, IEEE Trans. Ind. Inform. 15 (5) (2019) 3058-3067.

[25] G. Qiao, S. Leng, K. Zhang, Y. He, Collaborative task offloading in vehicular edge multi-access networks, IEEE Commun. Mag. 56 (8) (2018) $48-54$.

[26] H. Wang, X. Li, H. Ji, H. Zhang, Federated offloading scheme to minimize latency in mec-enabled vehicular networks, in: 2018 IEEE Globecom Workshops (GC Wkshps), IEEE, 2018, pp. 1-6. 
[27] X. Fan, T. Cui, C. Cao, Q. Chen, K. S. Kwak, Minimum-cost offloading for collaborative task execution of mec-assisted platooning, Sens. 19 (4) (2019) 847.

[31] M. Tehrani-Moayyed, F. Restuccia, S. Basagni, Comparative performance evaluation of mmwave $5 \mathrm{~g} \mathrm{nr}$ and lte in a campus scenario, Proceedings of IEEE VTC 2020 Fall.

[32] J. Feng, Z. Liu, C. Wu, Y. Ji, Mobile edge computing for the internet of

[34] A. B. Souza, J. Celestino, F. A. Xavier, F. D. Oliveira, A. Patel, M. Latifi, Stable multicast trees based on ant colony optimization for vehicular ad hoc networks, in: The International Conference on Information Networking 2013 (ICOIN), IEEE, 2013, pp. 101-106. 
[35] J. Härri, C. Bonnet, F. Filali, Kinetic mobility management applied to vehicular ad hoc network protocols, Comput. Commun. 31 (12) (2008) $2907-2924$.

[36] H. Menouar, M. Lenardi, F. Filali, Movement prediction-based routing (mopr) concept for position-based routing in vehicular networks, in: Vehicular Technology Conference, 2007. VTC-2007 Fall. 2007 IEEE 66th, IEEE, 2007, pp. 2101-2105.

[37] V. Namboodiri, L. Gao, Prediction-based routing for vehicular ad hoc networks, IEEE Trans. Veh. Technol. 56 (4) (2007) 2332-2345.

[38] J. Zhang, H. Guo, J. Liu, Y. Zhang, Task offloading in vehicular edge computing networks: A load-balancing solution, IEEE Trans. Veh. Technol. 69 (2) (2019) 2092-2104.

[39] Y. Sun, J. Song, S. Zhou, X. Guo, Z. Niu, Task replication for vehicular edge computing: A combinatorial multi-armed bandit based approach, in: 2018 IEEE Global Communications Conference (GLOBECOM), IEEE, 2018, pp. $1-7$.

[40] C. Chen, L. Chen, L. Liu, S. He, X. Yuan, D. Lan, Z. Chen, Delay-optimized v2v-based computation offloading in urban vehicular edge computing and networks, IEEE Access 8 (2020) 18863-18873.

[41] S. Shaham, M. Ding, M. Kokshoorn, Z. Lin, S. Dang, R. Abbas, Fast channel estimation and beam tracking for millimeter wave vehicular communications, IEEE Access 7 (2019) 141104-141118.

[42] M. Giordani, A. Zanella, T. Higuchi, O. Altintas, M. Zorzi, Performance study of lte and mmwave in vehicle-to-network communications, in: 2018 17th Annual Mediterranean Ad Hoc Networking Workshop (Med-Hoc-Net), IEEE, 2018, pp. 1-7. 
[43] T. Cui, Y. Hu, B. Shen, Q. Chen, Task offloading based on lyapunov optimization for mec-assisted vehicular platooning networks, Sens. 19 (22) (2019) 4974.

[44] S. Midya, A. Roy, K. Majumder, S. Phadikar, Multi-objective optimization technique for resource allocation and task scheduling in vehicular cloud architecture: A hybrid adaptive nature inspired approach, J. Netw. Comput. Appl. 103 (2018) 58-84.

[45] M. Chen, Y. Hao, M. Qiu, J. Song, D. Wu, I. Humar, Mobility-aware caching and computation offloading in 5g ultra-dense cellular networks, Sens. 16 (7) (2016) 974.

[46] G. Zobolas, C. D. Tarantilis, G. Ioannou, Exact, heuristic and metaheuristic algorithms for solving shop scheduling problems, in: Metaheuristics for scheduling in industrial and manufacturing applications, Springer, 2008, pp. 1-40.

[47] I. F. Akyildiz, C. Han, S. Nie, Combating the distance problem in the millimeter wave and terahertz frequency bands, IEEE Commun. Mag. 56 (6) (2018) 102-108.

[48] F. A. Teixeira, V. F. e Silva, J. L. Leoni, D. F. Macedo, J. M. Nogueira, Vehicular networks using the ieee 802.11 p standard: An experimental analysis, Veh. Commun. 1 (2) (2014) 91-96.

[49] G. F. Riley, T. R. Henderson, The ns-3 network simulator, in: Modeling and tools for network simulation, Springer, 2010, pp. 15-34.

[50] D. Krajzewicz, Traffic simulation with sumo-simulation of urban mobility, in: Fundamentals of traffic simulation, Springer, 2010, pp. 269-293.

1115 [51] J. Song, Y. Wu, Z. Xu, X. Lin, Research on car-following model based on sumo, in: The 7th IEEE/International Conference on Advanced Infocomm Technology, IEEE, 2014, pp. 47-55. 
[52] A. G. Howard, M. Zhu, B. Chen, D. Kalenichenko, W. Wang, T. Weyand, M. Andreetto, H. Adam, Mobilenets: Efficient convolutional neural networks for mobile vision applications, arXiv preprint arXiv:1704.04861 (2017) eprint.

[53] J. Redmon, A. Farhadi, Yolov3: An incremental improvement, arXiv preprint arXiv:1804.02767 (2018) eprint. 